\title{
FlyWire: Online community for whole-brain connectomics
}

\author{
Sven Dorkenwald ${ }^{1,2 *}$, Claire McKellar ${ }^{1 *}$, Thomas Macrina ${ }^{1,2 *}$, Nico Kemnitz ${ }^{1 *}$, Kisuk Lee ${ }^{1,5 *}$, Ran \\ $\mathrm{Lu}^{1 *}$, Jingpeng $\mathrm{Wu}^{1 *}$, Sergiy Popovych ${ }^{1,2}$, Eric Mitchell', Barak Nehoran ${ }^{1}$, Zhen Jia ${ }^{1,2}$, J. \\ Alexander $\mathrm{Bae}^{1,3}$, Shang $\mathrm{Mu}^{1}$, Dodam $\mathrm{Ih}^{1}$, Manuel Castro ${ }^{1}$, Oluwaseun Ogedengbe ${ }^{1}$, Akhilesh \\ Halageri $^{1}$, Zoe Ashwood ${ }^{1,2}$, Jonathan Zung ${ }^{1,2}$, Derrick Brittain ${ }^{4}$, Forrest Collman ${ }^{4}$, Casey \\ Schneider-Mizell ${ }^{4}$, Chris Jordan ${ }^{1}$, William Silversmith ${ }^{1}$, Christa Baker ${ }^{1}$, David Deutsch ${ }^{1}$, Lucas \\ Encarnacion-Rivera ${ }^{1}$, Sandeep Kumar ${ }^{1}$, Austin Burke', Jay Gager ${ }^{1}$, James Hebditch', Selden \\ Koolman ${ }^{1}$, Merlin Moore ${ }^{1}$, Sarah Morejohn ${ }^{1}$, Ben Silverman ${ }^{1}$, Kyle Willie ${ }^{1}$, Ryan Willie ${ }^{1}$, Szi-chieh \\ $\mathrm{Yu}^{1}$, Mala Murthy ${ }^{1} \dagger, \mathrm{H}$. Sebastian Seung ${ }^{1,2} \dagger$ \\ ${ }^{1}$ Princeton Neuroscience Institute, Princeton University, Princeton, NJ, USA \\ ${ }^{2}$ Computer Science Department, Princeton University, Princeton, NJ, USA \\ ${ }^{3}$ Electrical Engineering Department, Princeton University, Princeton, NJ, USA \\ ${ }^{4}$ Allen Institute for Brain Science, Seattle, WA, USA \\ ${ }^{5}$ Brain \& Cognitive Sciences Department, Massachusetts Institute of Technology, Cambridge, MA, USA \\ *These authors contributed equally \\ †Correspondence to sseung@princeton.edu and mmurthy@princeton.edu
}

\begin{abstract}
Due to advances in automated image acquisition and analysis, new whole-brain connectomes beyond $C$. elegans are finally on the horizon. Proofreading of whole-brain automated reconstructions will require many person-years of effort, due to the huge volumes of data involved. Here we present FlyWire, an online community for proofreading neural circuits in a fly brain, and explain how its computational and social structures are organized to scale up to whole-brain connectomics. Browser-based 3D interactive segmentation by collaborative editing of a spatially chunked supervoxel graph makes it possible to distribute proofreading to individuals located virtually anywhere in the world. Information in the edit history is programmatically accessible for a variety of uses such as estimating proofreading accuracy or building incentive systems. An open community accelerates proofreading by recruiting more participants, and accelerates scientific discovery by requiring information sharing. We demonstrate how FlyWire enables circuit analysis by reconstructing and analysing the connectome of mechanosensory neurons.
\end{abstract}




\section{INTRODUCTION}

Electron microscopy (EM) is the only technique that so far has proven capable of reconstructing all connections in a nervous system or brain. While the activity of large populations of neurons or even entire vertebrate brains (Ahrens et al. 2013) can be observed via calcium imaging, adult connectomes have so far been mapped for only one species, C. elegans (White et al. 1986; Cook et al. 2019). Fortunately, connectomes of more complex brains are now finally on the horizon. A major milestone in this direction has been the recent release of a Drosophila "hemibrain" connectome ( $\mathrm{Xu}$ et al. 2020). Part of a fly brain was imaged by EM, and automatically reconstructed using deep learning. Many errors in the reconstruction were corrected by 50 person-years of human "proofreading" to create a first draft of the hemibrain connectome.

The entire fly brain connectome would be of great scientific interest, because Drosophila melanogaster has become a popular model organism for circuit neuroscience. Flies are capable of a wide array of complex behaviors and functions, including social communication, aggression, spatial navigation, decision-making, and learning (Coen et al. 2014; Duistermars et al. 2018; Seelig and Jayaraman 2015; DasGupta, Ferreira, and Miesenböck 2014; Owald et al. 2015). While the hemibrain connectome is proving extremely useful for Drosophila circuit neuroscience, circuits that extend outside the hemibrain volume cannot be reconstructed (Extended Figure 1).

Therefore, we have created FlyWire, the first open online community for proofreading a connectome of a whole brain (flywire.ai). FlyWire is based on an EM dataset of a full adult fly brain (FAFB) that was previously released (Zheng et al. 2018). While FlyWire is dedicated to the fly brain, it introduces several new methods that will be generally applicable to whole-brain connectomics. The first is a novel data structure called the ChunkedGraph, which is the basis for proofreading. Like previous systems (J. S. Kim et al. 2014; Haehn et al. 2014; Knowles-Barley et al. 2016; Zhao et al. 2018), FlyWire represents neurons as connected components in a graph of supervoxels (groups of voxels). A naive implementation of this underlying data structure would scale poorly to large datasets. The ChunkedGraph divides the graph into spatially local chunks, and adds a hierarchy of extra vertices and edges to cache information about connected components. We show that edit operations are over an order of magnitude faster than systems relying on the naive implementation of the supervoxel graph. In addition, the ChunkedGraph enables real-time collaboration and stores the history of all edits.

FlyWire is also novel for its open social structure. Membership in the community is open to everyone. Community members immediately share the results of proofreading with each other. In contrast, members of the "walled garden" community, also reconstructing circuits from the FAFB dataset (Felsenberg et al. 2018; Dolan et al. 2018), are selected to avoid conflicts between labs working on the same circuit. Rather than restrict membership, FlyWire attempts to avoid conflicts by enforcing sharing of reconstructions with attribution. The hemibrain was reconstructed through a closed proofreading process that mobilized paid workers, and updated results are released to the public as the internal proofreading progresses (Xu et al. 2020). Our 
principle of openness was inspired by a previous project to reconstruct larval Drosophila circuits (A. Cardona, personal communication).

The walled garden has historically used fully manual skeletonization to reconstruct neural circuits from FAFB (Felsenberg et al. 2018; Dolan et al. 2018). Since manual skeletonization is laborious, the walled garden is starting to migrate to semi-automated reconstruction (Z. Zheng et al. 2020) based on piecing together automatically generated skeletons (Li et al. 2019). FlyWire, in addition to being open, enables true 3D interactive proofreading of a volumetric segmentation.

A final novelty of FlyWire is that the accuracy of the automated reconstruction was boosted by realigning the serial section images using deep learning (Mitchell et al. 2019). In the published FAFB dataset, which was aligned with conventional computer vision algorithms (Zheng et al. 2018), misalignments were numerous enough to be the dominant failure mode for automated reconstruction.

We show that most neurons in FlyWire can be reconstructed within 1.5 hours to a degree that is sufficient for morphological and circuit level analyses. Using FlyWire we produced the first complete connectivity diagram between known early mechanosensory/auditory neurons and discovered previously unknown connection patterns, including cross-hemispheric connections likely to be important for sound localization. As further evidence of FlyWire's utility, it was also recently used to map the connectivity of Drosophila neurons related to a persistent internal state (Deutsch, Pacheco, and Encarnacion-Rivera 2020).

Given recent progress in automated EM image acquisition (Yin et al. 2019) reconstruction (Jain, Seung, and Turaga 2010; Lee et al. 2019; Januszewski et al. 2018), we can expect many other large scale connectome projects in the future. The methods introduced by FlyWire should be generally useful for these projects.

\section{RESULTS}

\section{Neuron segmentation}

We realigned the serial section images of the FAFB dataset (Zheng et al. 2018), and generated an automated segmentation (see Methods, Extended Data Fig. 2 for a quality assessment of the realignment). The automatically generated segments often show many or all of the expected parts of a fly neuron: a soma, dendrites, axon terminals, and a primary neurite (the usually unbranched proximal neurite connecting the soma to branching arbors downstream).

We examined reconstructions of well known cell types before and after proofreading to assess segmentation quality and test whether neurons can be identified before proofreading (Fig. 1). Indeed, the automated segmentation is often highly accurate to begin with (quantification below) and unique morphological features across the examined cells are visible without proofreading. Qualitative comparison between images of light-level stains of the giant fiber neurons (Pézier et al. 2016) (Fig. 1 a,c,e) and a mushroom body APL neuron (Wu et al. 2011) (Fig. 1 b,d,f) show that our semi-automated segmentation procedures are able to capture neurons in their entirety. 

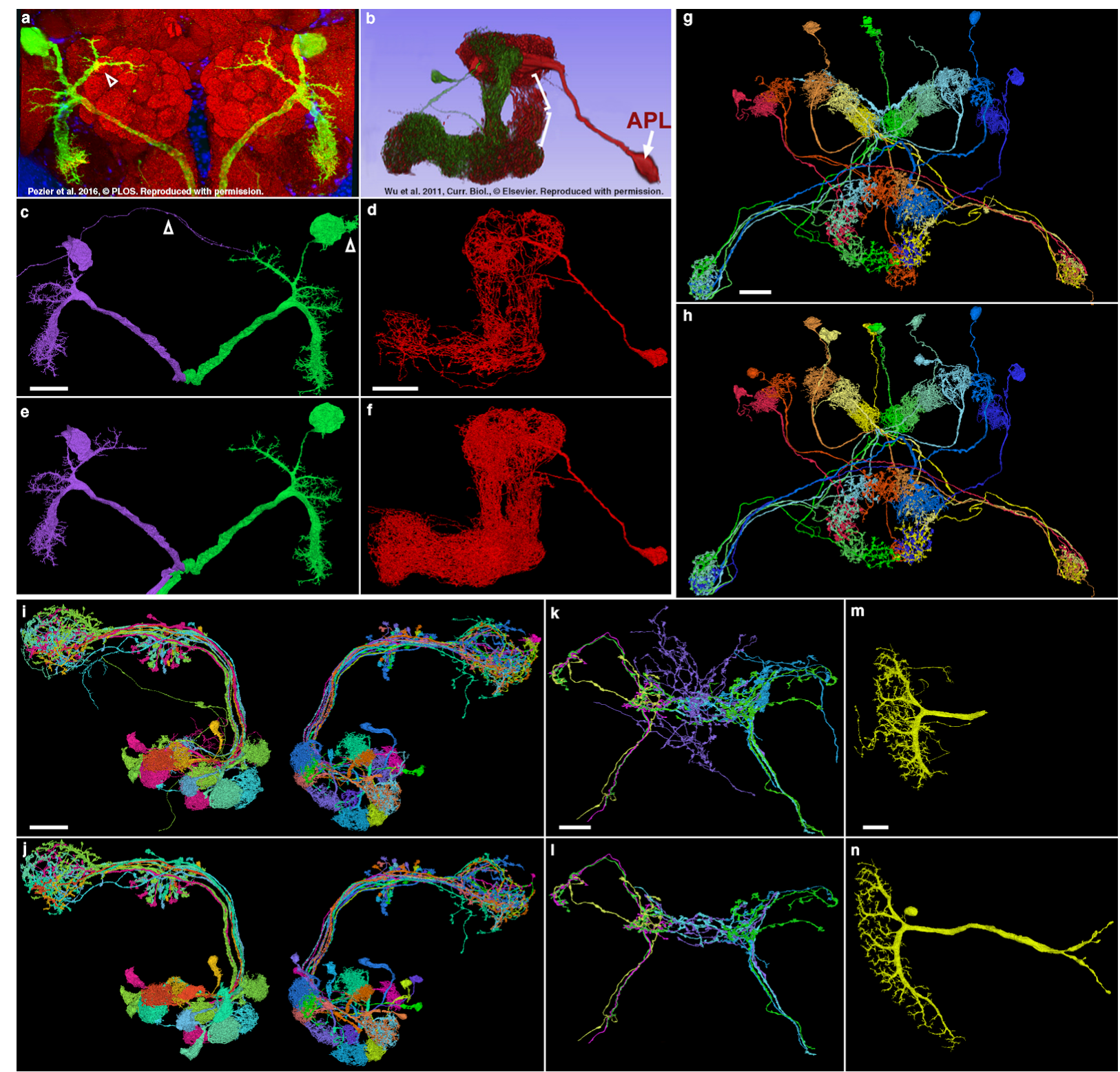

Figure 1. Assessing segmentation quality using known neurons. (a-d) Comparison of light-level stains of known giant fiber neurons (a) and a mushroom body APL neuron (b, red) to FlyWire's Al-predicted segmentation of these cells $(\mathbf{c}, \mathbf{d})$. (e,f) The same neurons shown following proofreading. (g-n) Examples of other known cell types before and after proofreading (top and bottom of each pair, respectively): central complex neurons ( $\mathbf{g}, \mathbf{h})$, olfactory projection neurons $(\mathbf{i}, \mathbf{j})$, gustatory receptor neurons $(\mathbf{k}, \mathbf{l})$ and a lobula plate tangential cell $(\mathbf{m}, \mathbf{n})$. All views frontal except APL and central complex neurons: dorso-frontal view. Scale bars: (c, d, e, f, i, j) $30 \mu \mathrm{m},(\mathrm{g}, \mathrm{h}, \mathrm{k}, \mathrm{l}) 15 \mu \mathrm{m},(\mathrm{m}, \mathrm{n}) 20 \mu \mathrm{m}$ 
a

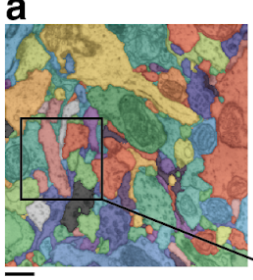

b

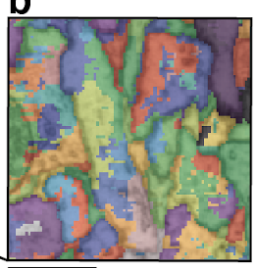

C

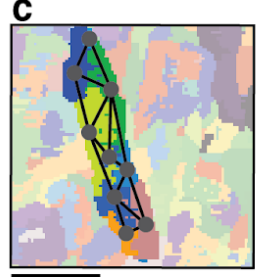

d

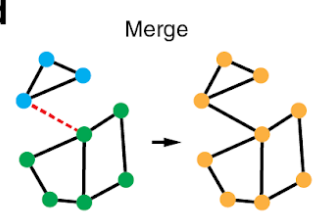

e

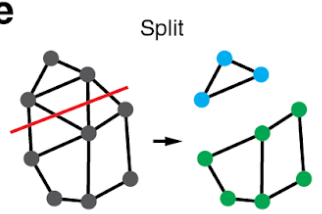

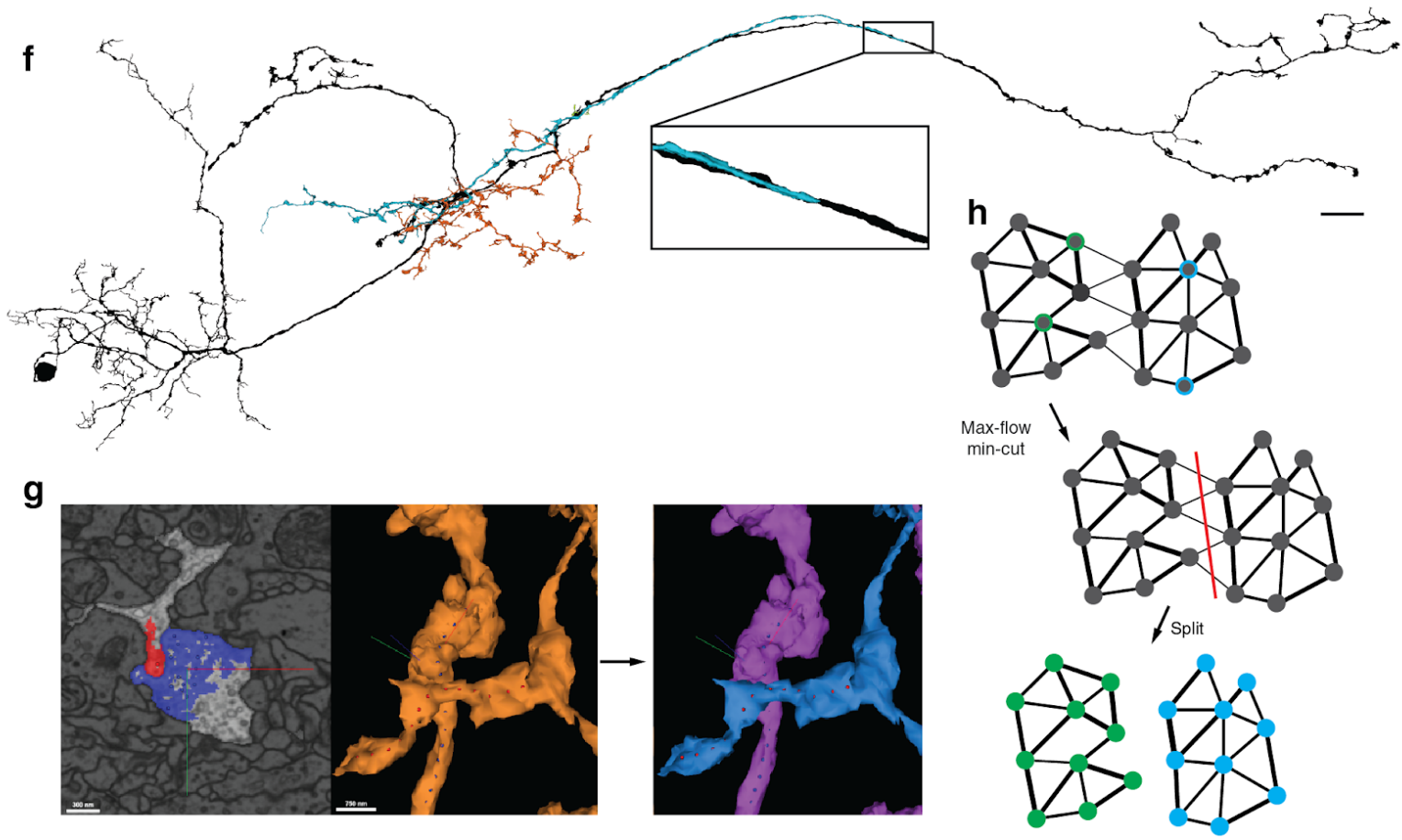

Fig. 2 Proofreading the supervoxel graph. (a) Automated segmentation overlaid on the EM data. Each color represents an individual putative neuron. (b) Each component in (a) is made up of many supervoxels shown in different colors. (c) This panel corresponds to the framed square in (a) and the full panel in (b) where we highlighted the supervoxels belonging to a particular neuron and overlaid a cartoon of the supervoxel graph. (d) Touching supervoxels (circles) may be connected through edges in the graph indicating that they belong to the same connected component (solid lines). Merge operations add edges between supervoxels resulting in new neuronal components (orange). (e) Split operations remove edges resulting in new neuronal components (green, blue). (f) Example neuron after proofreading (black). Green, blue and red components were removed during proofreading (see Ext. Data Fig. 4 for the added pieces). While edit operations have global effects, the edits to the supervoxel graph themselves are performed at a local level. (g) For splits, users place points (red and blue dots) either in 2D or 3D (center panel) that are linked to the underlying supervoxels (left panel). The proofreading backend then automatically determines which edges need to be removed and performs the split (right panel). (h) For the operation shown in (g) the backend performs max-flow min-cut on the local supervoxel graph to determine the optimal cut that separates the user-defined input locations (green and blue framed circles). The thickness of the edges symbolizes the edge weight (cartoon). Scale bars $(a, b, c): 1 \mu \mathrm{m}(\mathrm{f}): 10 \mu \mathrm{m}$ 


\section{Chunked supervoxel graph as data structure for proofreading}

Proofreading consists of two basic operations: merging falsely disconnected segments and splitting falsely merged ones. For efficient editing of the automatically generated segments, we represent the segmentation as a supervoxel graph. Each graph node is a supervoxel, an atomic group of voxels that is never split (Fig. 2a,b). At any moment in time, the current segmentation is represented by the connected components of the supervoxel graph (Fig. 2c). Two segments can be merged into one by adding an edge to the graph (Fig. 2d), and one segment can be split into two by removing edges (Fig. 2e,f). In order to make splits easy, users can place points on both sides of a proposed split (Fig. 2g) and our proofreading system identifies the edges that need to be removed to separate them. Our system deploys a max-flow min-cut algorithm operating on a local cutout of the supervoxel graph using predicted edge weights as capacities (Fig. $2 \mathrm{~h}$ ).

Scaling proofreading to a community demands that all users can access the latest state of the segmentation and that multiple users can work on the same neuron without introducing inconsistencies. To allow interactive and continuous proofreading, edits must be resolved quickly and visuals must be updated for the user. At the same time, older states of the segmentation must be accessible for review and publications. However, reads, writes and computations on the supervoxel graph can be time-consuming, because they scale at least linearly with the size of the components. That is because edits have global effects on the connected components even though they only introduce local changes (Fig. 2f). Because of these challenges, no system for community based proofreading of entire neurons exists that scales to datasets as large as FAFB. Existing systems on smaller datasets restrict what proofreaders can work on(J. S. Kim et al. 2014) or do not allow open proofreading by a community (Xu et al. 2020).

We designed a novel data structure, the ChunkedGraph, to address these challenges. The ChunkedGraph leverages the fact that edits only change a small region of a neuron, leaving the rest unchanged. It caches information about connected components spatially allowing it to update components rapidly after edits and restricts the part of the graph that needs to be read and analyzed for them. For this, the nodes of the supervoxel graph are divided into spatial chunks (Extended Data Fig. 3). A supervoxel spanning chunk borders is carved into multiple supervoxels, each contained within a chunk. Each chunk also stores edges between the supervoxels in that chunk. We build an octree on top for storing the connected component information (Fig. 3b). In this tree, nodes in higher layers, abstract nodes, represent connected components in the spatially underlying graph (Fig. 3b-d). Because the ChunkedGraph decouples regions of the same neuron from each other, regions unaffected by an edit do not need to be read and included into calculations, and changes only need to propagate up the tree hierarchy (Fig. 3c, Extended Data Fig. 4). Each segment is a tree, and the ChunkedGraph is a forest of all the segments.

The ChunkedGraph is initialized by ingesting the initial supervoxel graph created by our automated segmentation pipeline (Dorkenwald et al. 2019). Our pipeline creates supervoxels by grouping voxels that belong to the same cell with very high confidence, according to the affinity predicting neural network. Edges are added to the ChunkedGraph for every pair of 
a

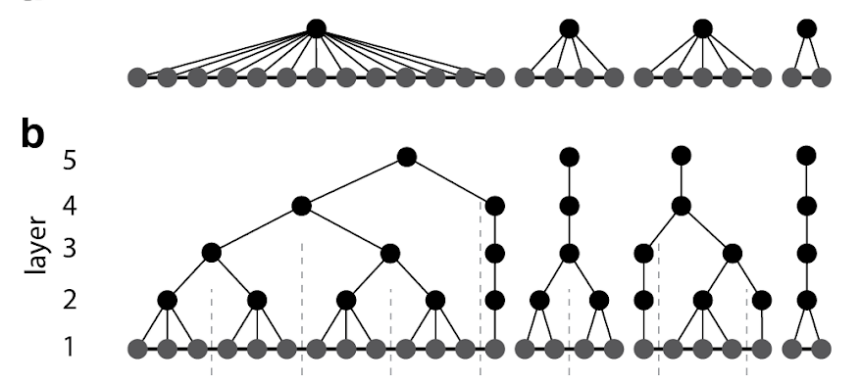

C 5
5
4
$\stackrel{\bar{\Xi}}{3} 3$
2
1
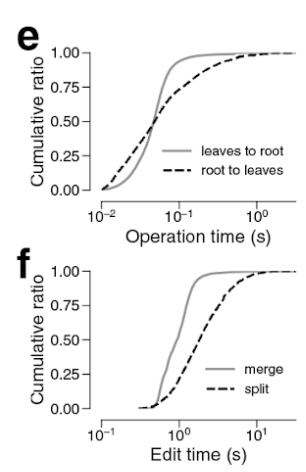

$t_{1}=t_{2}>t_{0}$

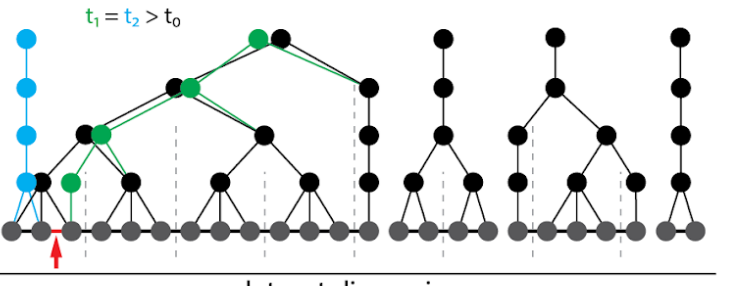

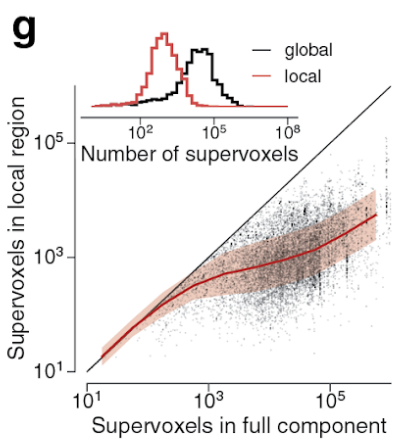

d

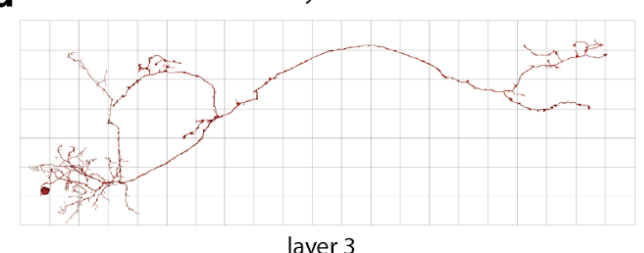

layer 3

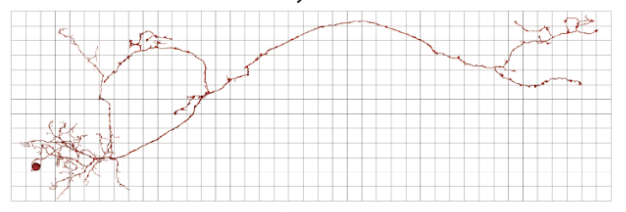

layer 2

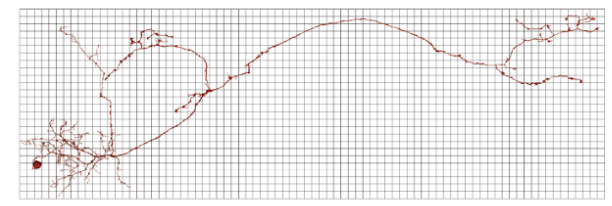

h

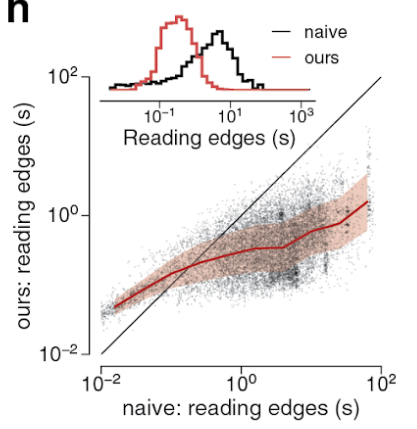

i

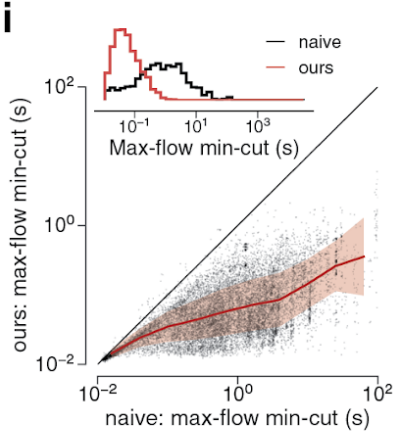

Fig. 3 The ChunkedGraph approach for proofreading supervoxel graphs. (a) One-dimensional representation of the supervoxels graph. In the simplest approach (naive), one would store the connected component information (neuronal component) in a dedicated parent node. (b) We propose an alternative data structure to enable proofreading of very large supervoxel graphs. We store the connected component information in an octree structure where each abstract node (black and colored nodes in levels $>1$ ) represents the connected component in the spatially underlying graph (dashed lines represent chunk boundaries, see Ext. Fig. 3). Nodes on the highest layer represent entire neuronal components. (c) Edits to the ChunkedGraph (here, a split; indicated by the red arrow and removed red edge) do not require the loading of the entire supervoxel graph to recompute the neuronal connected components. We can reuse the connected component information stored in the abstract nodes that did not see a change to their underlying graph. (d) Chunks double in size along each dimension when going to the higher layer. (e) Server response times for the remapping of the connected components from root to supervoxel $(\mathrm{N}=3,080,494)$ and supervoxel to root $(\mathrm{N}=12,096)(\mathrm{f})$ as well as splits $(\mathrm{N}=2,497)$ and merges $(\mathrm{N}=4,612)$ for real user interactions in the beta-phase of FlyWire. (g) Compared to the naive approach (Fig. 3a), our approach reduces the number of supervoxels that need to be loaded for a split (global vs local) (h) The subgraph can be read faster and (i) speeds up max-flow min-cut calculations (Fig. 2g,h). The red lines in (g, h, i) are mean and the shaded area standard deviation of bins along $x$-axis ( 10 bins); $\mathrm{N}=15,233$. 
neighboring supervoxels in the same segment. Edge weights are also available from the automated segmentation pipeline, and are also ingested into the ChunkedGraph. Proofreading starts from this initial condition, and proceeds by adding and subtracting edges from the ChunkedGraph.

\section{Visualization of segments in 2D and 3D}

FlyWire provides several visualizations to enable the user to find and correct segmentation errors. 2D cross sections of the EM image are displayed in grayscale. Three orthogonal cross sections are available. 2D cross sections of the segmentation are displayed in color, and can be overlaid on the EM images (Extended Data Fig. 5a, left). FlyWire also displays a 3D rendering, a mesh, of user-selected segments (Extended Data Fig. 5a, middle). All of these visualizations utilize Google's Neuroglancer software (Maitin-Shepard 2020), which enables viewing of volumetric images in a web browser.

When a user interactively selects a supervoxel with a mouse click, the system rapidly displays all supervoxels belonging to the same segment within the field of view of the user. This is accomplished by searching the ChunkedGraph as follows. The search first traverses the tree from the selected supervoxel to the root node at the top level of the hierarchy. For mapping supervoxel to root, the server responded with a median time of $47 \mathrm{~ms}$ and 95th percentile of 111 ms (Fig. 3e, $n=12,096$ ). Once the search has reached the root, it proceeds back down the tree to identify all supervoxels connected to it within the displayed area, making use of the octree structure of the ChunkedGraph. For mapping root to supervoxels, the server responded with a median time of $48 \mathrm{~ms}$ and 95th percentile of $465 \mathrm{~ms}$ per displayed chunk $(n=3,080,494)$. Such fast response times are crucial for a globally distributed system if every user is to see the latest state of the segmentation and no data is stored locally. The above times are server response times and were measured during FlyWire's beta phase (graph with 2.38 billion supervoxels).

\section{Proofreading by editing the supervoxel graph}

Interactive proofreading (Fig. $2 \mathrm{~g}$ ) is implemented using the ChunkedGraph as follows. The user specifies a merge operation by selecting two supervoxels with mouse clicks. An edge between this pair is added to the supervoxel graph (Fig. 2d, Extended Data Fig. 5). Merge edits took 940 $\mathrm{ms}$ at median, and $1841 \mathrm{~ms}$ at 95th percentile ( $\mathrm{n}=4,612)$ (Fig. 3f). The user specifies a split operation by selecting supervoxels with mouse clicks (Fig. 2e,g). The system applies a min-cut algorithm to remove a set of edges with minimum weight that leaves the two supervoxels in separate segments (Fig. $2 \mathrm{~g}, \mathrm{~h}$ ). Split edits had a median time of $1,818 \mathrm{~ms}$, and 95th percentile time of $7,137 \mathrm{~ms}(\mathrm{n}=2,497)$ (Fig. 3f).

After each edit, the ChunkedGraph generates new abstract nodes in higher layers $(>1$, colored nodes in Fig. 2c and Extended Data Fig. 4a). Here, the tree is only traversed in its height and not its width because connected components in neighboring regions are cached in abstract nodes. We use the same abstraction for fast mesh generation of new components by restricting the application of costly and slow meshing algorithms (eg. marching cubes) to single chunks. We only compute meshes from the segmentation for abstract nodes on level 2 (Extended Fig. 3d) 
and then stitch these to larger components according to the hierarchy such that each abstract node up to a predefined layer has a corresponding mesh. The ChunkedGraph dynamically generates instructions for which mesh files to load for a given component.

We compared the performance of the ChunkedGraph versus an equivalent naive implementation of the supervoxel graph (Methods) (Fig. 3h,i). We measured two different parts of split operation: reading of the edges to compute the split and the min-cut algorithm. The ChunkedGraph greatly benefits from being able to restrict the operations to a subregion (Fig. $3 \mathrm{~g}$ ), leading to orders of magnitude faster reading and calculations (Fig. 3h,i). The ChunkedGraph incurs a minor overhead that is only notable for very small components.

The ChunkedGraph allows concurrent and unrestricted proofreading by many users through serializing edits on a per-neuron level. Edits generate new, timestamped nodes on higher levels (Fig. 3c, Extended Data Fig. 4b), allowing the retrieval of any older state of the segmentation by applying a time filter during tree traversal. Edits can only be applied to the latest version of the segmentation. We implemented the ChunkedGraph with Google's BigTable (Chang et al. 2008), a low-latency NoSQL database, making use of its timestamping features and atomic row write and read capabilities. For the user, the ability to view the state of a cell from any past time point in the proofreading process is helpful for reviewing one's own work or the work of others. This is analogous to viewing past versions of a Wikipedia article, which are recreated using the edit history (Priedhorsky et al. 2007) (Fig. 4a).

\section{Extracting synaptic connections}

With hundreds of millions of synaptic connections in the fly brain (Buhmann et al. 2019), automated synaptic partner identification is required to facilitate connectivity analysis at scale. Several methods have been proposed for synapse detection in large EM datasets (Heinrich et al. 2018; Staffler et al. 2017; Dorkenwald et al. 2017; Huang, Scheffer, and Plaza 2018; Buhmann et al. 2019; Turner et al. 2019) but only a few solved the unique problem of partner assignments in polyadic synapses in the fly (Buhmann et al. 2018; Buhmann et al. 2019; Huang, Scheffer, and Plaza 2018; Kreshuk et al. 2015). FlyWire is compatible with any existing and future method that identifies synaptic partners and their pre- and postsynaptic sites. Currently, only Buhmann et al.(Buhmann et al. 2019) demonstrated their method on the whole fly brain. We imported their synapses into our realigned coordinate space and made them available to the community.

A fly neuron is said to consist of a thicker, microtubule-rich "backbone" and numerous thin "twigs" (Schneider-Mizell et al. 2016) (Fig. 4a). The distinction can be somewhat subjective in borderline cases, but is quite useful in practice. The automated segmentation contains many small "orphan twigs" that are not assigned to any large neuronal object. Attaching orphan twigs is time consuming and difficult because twigs contain very thin processes. Therefore, our proofreaders did not correct orphan twigs for the most part. The hemibrain similarly contains many orphan twigs after proofreading (Xu et al. 2020). 


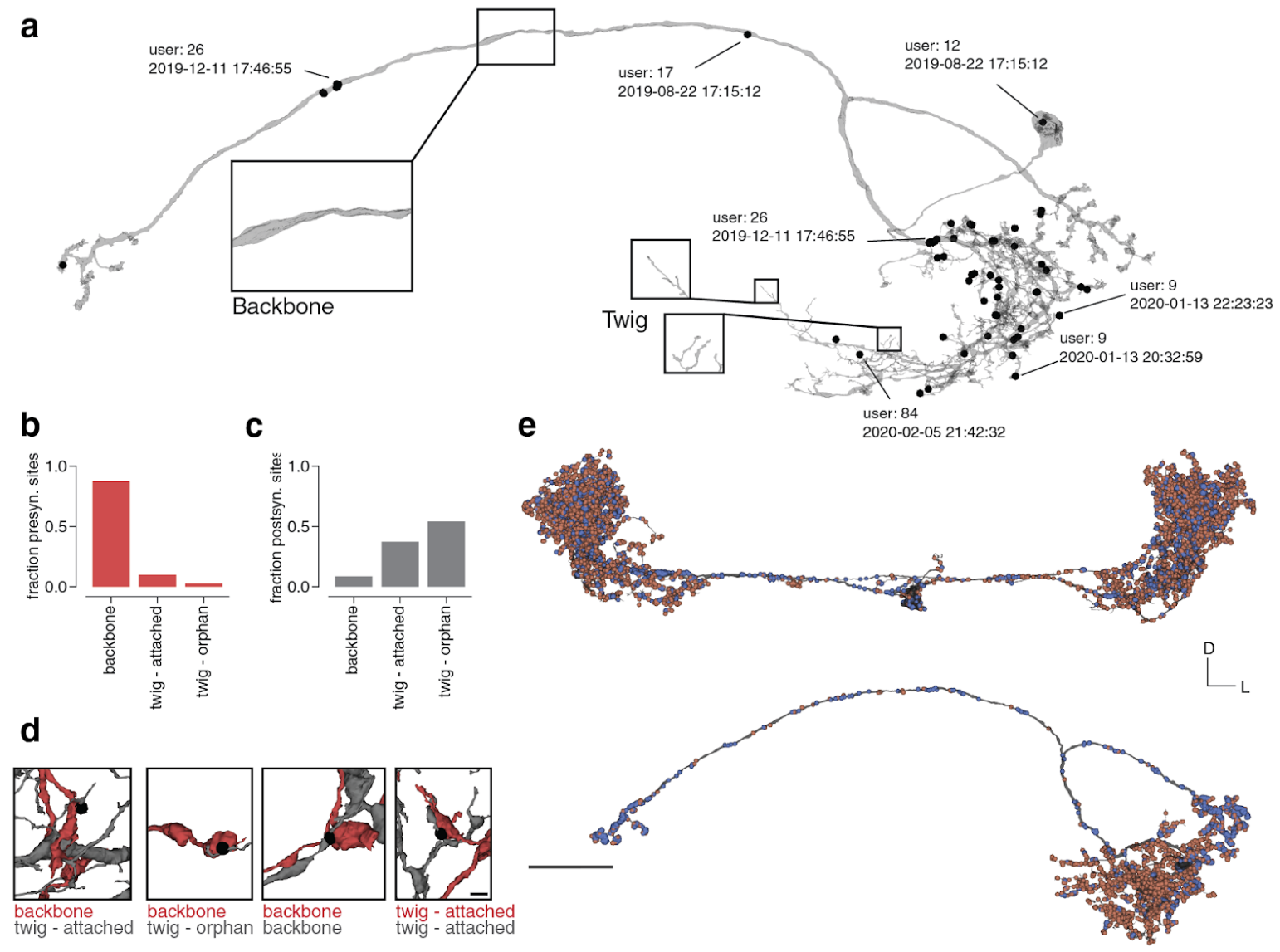

Figure 4. Attaching automatically detected synapses to neurons. (a) Each edit (black dot) is linked to a user and timestamp enabling the retrieval of the edit history and credit assignment post-hoc. Neurons in the fly brain can be roughly partitioned into sections known as "twigs" and "backbone". (b) We classified pre- and (c) post-synaptic segments based on their morphology and whether they are attached to a bigger component that will be attached during a conservative procedure. (d) shows examples of these assessments. (e) shows a proofread WV-WV PN (top) and AMMC-B1 (bottom) with automatically detected synapses attached (blue: presynaptic, red: postsynaptic). Scale bar: (d): $1 \mu \mathrm{m}$; (e) $25 \mu \mathrm{m}$
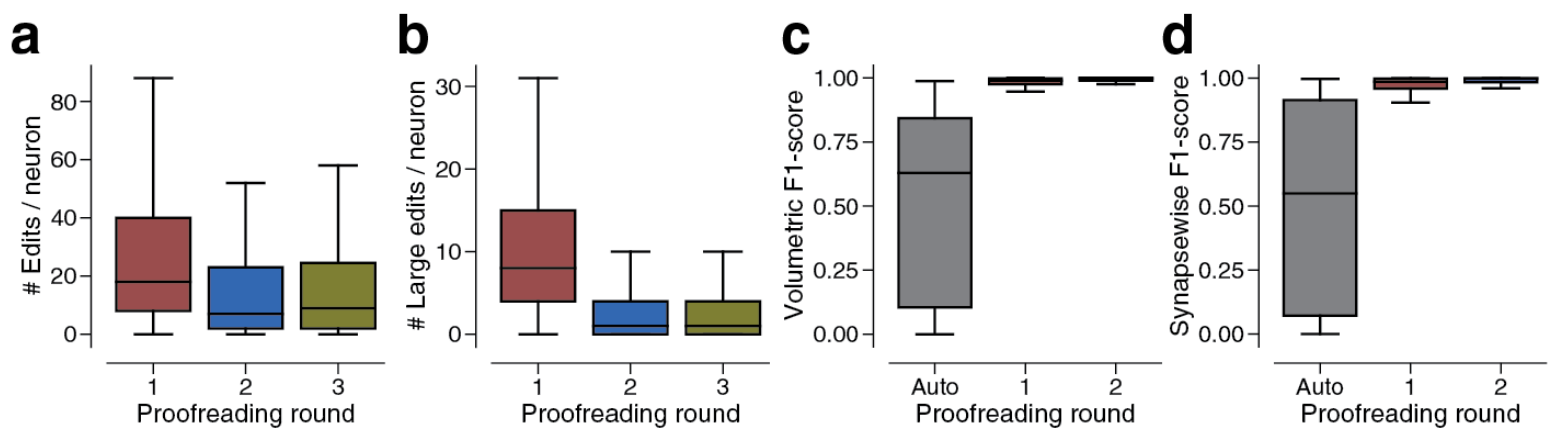

Figure 5. Proofreading in FlyWire. (a) Number of edits per neuron ( $\mathrm{N}=183)$ and proofreading round. (b) Same as (a) but restricted to large edits $\left(>1 \mu \mathrm{m}^{3}\right)$. Large edits are usually edits on "backbones" whereas small edits tend to affect "twigs". (c) Comparing the F1-Scores (0-1, higher is better; with respect to proofreading results after three rounds) between different proofreading rounds according to volumetric completeness and (d) assigned synapses. "Auto" refers to reconstructions without proofreading. 
Avoiding the excessive labor of reattaching twigs comes at some cost: synapses involving orphan twigs will be missing from the reconstruction. Fortunately, many fly neurons are redundantly connected, with up to hundreds of synapses possible between a connected pair (Takemura et al. 2013). It has been proposed that a neuron pair sharing fewer than 10 synapses represents a minor connection (Meinertzhagen 2018). If omissions of synapses are statistically independent, then connections will be recalled with a probability that increases with the number of synapses involved (Schneider-Mizell et al. 2016).

We quantified the number of missing synapses due to orphan twigs by evaluating the segmentation at 612 randomly picked synaptic locations from Buhmann et al. (see Methods). For each of these synapses an expert judged whether the pre- and postsynaptic reconstructions were at a backbone or twig and whether the twig was attached ("twig - attached") to a backbone or orphan ("twig - orphan") (Fig. 4b-d, see Methods). We found that $40.6 \%$ of all postsynaptic and $78.2 \%$ of presynaptic twigs were attached to backbones. In aggregate, we expect our proposed conservative proofreading to at least include all "backbone" and "twig - attached" segments in a proofread neuron. Our assessment leads to a combined estimate of $44.6 \%$ of synapses with pre- and postsynaptic segments attached on average after proofreading. In practice, major connections (>9 synapses, $99.7 \%$ with at least one synapse) and most minor connections with at least 3 synapses are maintained (83\% with at least one synapse) due to the redundant nature of connections in the fly brain (Schneider-Mizell et al. 2016; Meinertzhagen 2018).

For analysis, we assign synapses to neurons based on their pre- and postsynaptic coordinates (Fig. 4e). Since proofreading changes the assignment of synapses to neurons, we continuously release new versions of the synapse table.

\section{Quantification of proofreading effort and accuracy}

To assess effort required to proofread neuronal backbones, we proofread 183 neurons mostly with projections in early mechanosensory neuropils (antennal mechanosensory and motor center (AMMC), wedge (WED), and ventrolateral protocerebrum (VLP)). Each neuron was proofread by three different people in successive rounds. The number of errors corrected decreased after the first round (Fig. 5a), most notably large corrections (volumetric difference > $1 \mu \mathrm{m}^{3}$ ) decreased from a median of 8 in the first round to medians of 1 and 0 in the second and third round (Fig. 5b).

To quantify the impact of the different proofreading rounds further, we scored the reconstructions after each round with respect to their state after the third round. We calculated F1-Scores with respect to volumetric completeness and correct synapse assignments (pre- and postsynaptic irrespectively) (Fig. 5c,d). One round of proofreading was enough to recover an accurate morphology and synapse assignment in most cells (median F1-Scores of .99 for both volumetric and synapse based assessments). We measured times for 49 of the 183 neurons and found that first-round proofreading took a median of 90 minutes per neuron (mean: 135 minutes). 

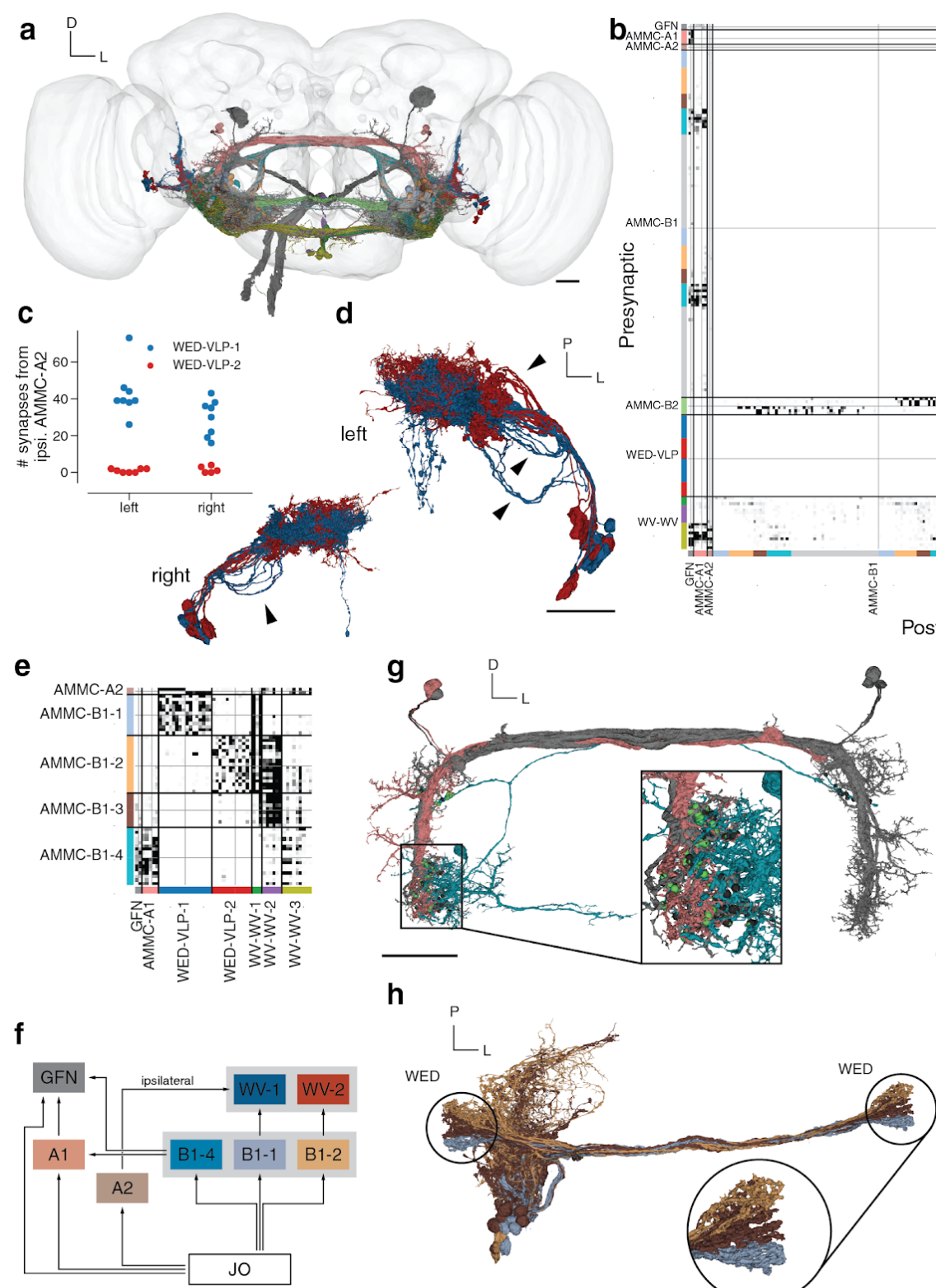

h
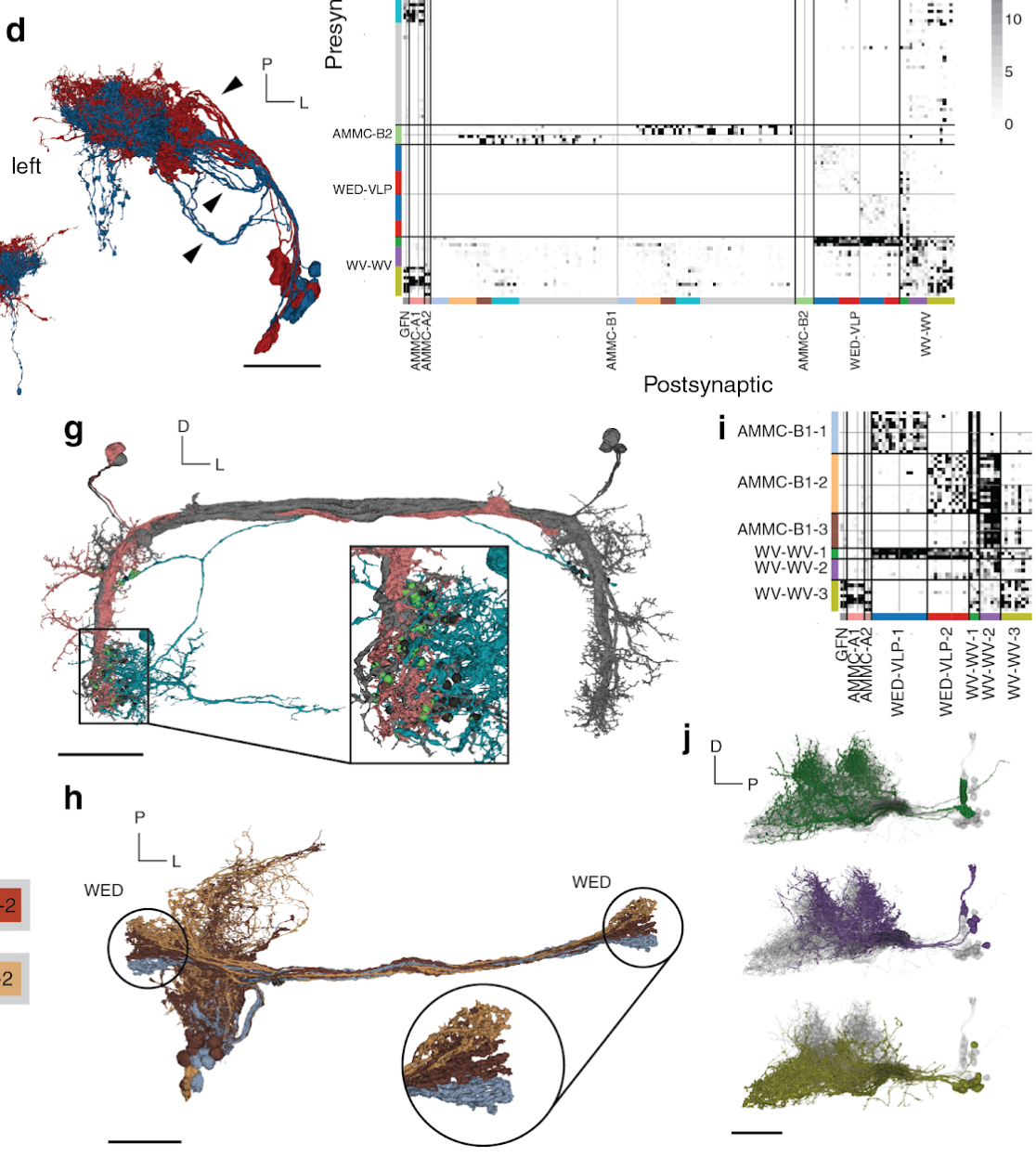

d left
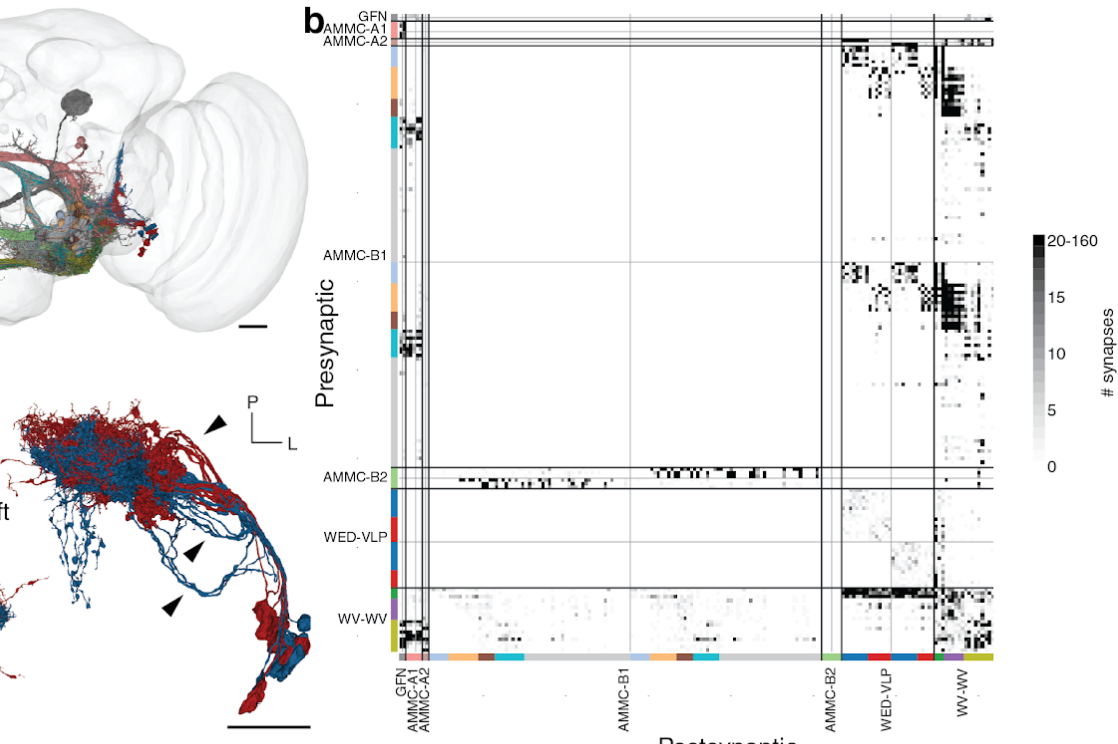

Postsynaptic

Figure 6. Auditory connectivity extracted with FlyWire. (a) We included 180 neurons from both hemispheres into our analysis, here colored by their cell type (black: giant fiber neuron, turquoise: AMMC-A1, orange: AMMC-A2, dark blue: AMMC-B1, light blue: AMMC-B2, red: WED-VLP PN, green: WV-WV PN). (b) Connectivity diagram between all 180 neurons ordered by cell type. Gray through lines divide cells from different hemispheres (left/top: left hemisphere, right/bottom: right hemisphere) and colored bars separate putative sub-celltype (see Extended Data Fig. 6 for matrices with different colormap cutoffs). (c) Analyzing the synaptic inputs of the WED-VLP neurons onto the suggested groups shows differential input from AMMC-A2 subtypes. (d) The two WED-VLP groups differ in their morphology (see arrow heads). (e) We grouped AMMC-B1 neurons according to their outputs on to other cell types in the AMMC. (f) Abridged summarized connectivity diagram showing the selective targeting of WED-VLP PNs (WV) by AMMC-B1s (B1) and the cross-pathway innervation by AMMC-B1-4s. (g) The connectivity based groupings of AMMC-B1 neurons correspond to a stration of axonal arbors in both hemispheres (arrow heads). (h) One group of AMMC-B1 neurons targets AMMC-A1 neurons in both hemispheres (red: AMMC-A1, turquoise: AMMC-B1-4, gray: all AMMC-A1s). We automatically detected 217 synapses from this specific AMMC-B1 neuron onto all AMMC-A1 neurons (balls), 87 of which on the shown AMMC-A1 (green balls). (i) Commissural neurons vary in their connectivity with other cell types. (j) The suggested subgrouping correlates with collocations of their cell bodies as well as different arborizations. Scale bars: $25 \mu \mathrm{m}$ 
Researchers can use FlyWire to proofread to their desired level of accuracy and some researchers have reported scientific benefits without any proofreading at all. For others it may be sufficient to proofread backbones, as in Deutsch et al. (Deutsch, Pacheco, and Encarnacion-Rivera 2020) using FlyWire to reconstruct a circuit involved in female social behavior.

\section{Novel connections and subtypes in mechanosensory pathways}

To validate FlyWire as a circuit discovery platform, we proofread and analyzed 180 neurons (belonging to seven major types) in the AMMC, WED, and VLP neuropils (Fig. 6a), all previously shown to respond to mechanosensory stimuli, such as sound or wind (Tootoonian et al. 2012; Lai et al. 2012; Vaughan et al. 2014; Yamada et al. 2018). These neurons were found based on their previously identified morphology and cell body location (Kamikouchi et al. 2009; Lai et al. 2012; Tootoonian et al. 2012) (see Methods, and Supplementary Table 1 for numbers of neurons per cell type).

Airborne mechanosensory stimuli activate Johnston's Organ (JO) of which distinct populations send broadly tonotopic projections to five different zones within the AMMC. AMMC neurons in turn connect with the WED and VLP (Kamikouchi et al. 2009). We identified neurons proposed to have dendrites in AMMC zones $A$ (AMMC-A1, AMMC-A2, GFN) and B (AMMC-B1, AMMC-B2) and therefore receive inputs largely from JO-As and JO-Bs respectively $(\mathrm{H}$. Kim et al. 2020). Although prior work identified only 10 AMMC-B1 neurons per hemisphere $(\mathrm{H}$. Kim et al. 2020; Lai et al. 2012), we identified 61 neurons in the left hemisphere and 58 neurons in the right hemisphere, all with clear B1 morphology (Extended Data Fig. 6). We additionally identified neurons belonging to cell types WED-VLP (aka iVLP-VLP (Lai et al. 2012)) and WV-WV (aka iVLP-iVLP (Lai et al. 2012) or WED-WED (Clemens et al. 2015)).

AMMC-B1 neurons respond strongly to frequencies present in conspecific courtship songs (Azevedo and Wilson 2017) and are thought to target WED-VLPs, based on the proximity of their processes (Lai et al. 2012), forming a putative pathway for courtship song processing in the brain. GFNs and AMMC-A1 neurons on the other hand, while responsive to song stimuli (Tootoonian et al. 2012; Clemens et al. 2015), are core components of the Drosophila escape pathway (von Reyn et al. 2014; Allen et al. 2006). We determined if there was any overlap between these two pathways and also looked for important subtypes, based on connectivity and morphology, within each cell type. Finally, as FlyWire enables identification of synaptic connections across both brain hemispheres, we found pathways that could mediate the detection of sound or wind direction via cross-hemispheric connections. To do this, we created a wiring diagram between all 180 identified neurons by combining our reconstructions (Fig. 6a) with the synapses from Buhmann et al. (Buhmann et al. 2019) with minor additional synapse proofreading (Fig. 6b, see Ext. Data Fig. 7 for a matrix ordered by subtype and a matrix with different colormap threshold, see Methods).

Our analysis confirms previously proposed pathways between AMMC-A1 and GFN (Phelan et al. 2008) as well as AMMC-B1 and WED-VLPs (Lai et al. 2012). However, we found that only a 
minority of the AMMC-B1 neurons innervated WED-VLPS (left: 15/61, right: 14/58, Supplementary Table 1, Fig. 6b) and that two subgroups of AMMC-B1s targeted two subgroups of WED-VLPS. This partition of WED-VLPs was directly related to input from ipsilateral AMMC-A2s (Fig. 6c) and a morphological separation of their arbors (Fig. 6d). It stands out that WED-VLP-1 neurons receive convergent input from AMMC-A2 and AMMC-B1-1 neurons, positioning them to encode both auditory stimulus motion energy and sound vibration (Azevedo and Wilson 2017).

AMMC-B1 neurons can be divided into at least 5 subtypes (see Extended Data Fig. 6 for a morphological comparison and Supplementary Table 2 links to the data) - AMMC-B1-1 and AMMC-B1-2 neurons project to WED-VLP neurons, AMMC-B1-3 neurons target only the WV-WV neurons, and AMMC-B1-4 neurons send outputs (with up to 90 synapses per connection) to the GFN and AMMC-A1 neurons, suggesting the existence of (previously unknown) feedback from the courtship song processing pathway to the escape pathway (Fig. 6h). AMMC-B1-u (u for unidentified) neurons had almost all of their synapses onto neurons not included in our set of 180 neurons. When validating these subtypes morphologically, we found that the axonal arbors of AMMC-B1-1, -2 and -3 striate the WED in both hemispheres (Fig. $6 \mathrm{~g}$ ). AMMC-B2 neurons receive input from ipsilateral JO-B neurons, are GABAergic, and proposed to sharpen the tuning of AMMC-B1 for sound frequencies (Yamada et al. 2018); we found that they only target AMMC-B1 neurons in the contralateral hemisphere (Fig. 6b, Extended Fig. 1a), suggesting a role in spatial localization of sounds.

WV-WVs are GABAergic (Lai et al. 2012) with cell bodies in the center of the brain and symmetrical processes in both hemispheres - these neurons are therefore well positioned to provide feedback inhibition within the circuit. We identified a subgroup that targets GFN, AMMC-A1 and AMMC-A2 neurons in both hemispheres (WV-WV-3) as well as a subgroup that strongly synapses onto WED-VLPs (WV-WV-1). Lastly, we identified a group (WV-WV-2) receiving input predominantly from $A M M C-B 1-2$ and AMMC-B1-3 neurons but not from AMMC-B1-1 neurons (Fig. 6i). That WV-WV-3s receive input from AMMC-B1-4 neurons and contact GFN and AMMC-A1 neurons is again consistent with cross-talk between the courtship song and escape pathways. When observing the morphology of these three types of WV-WV neurons, we found a correlation with the location of cell bodies and arborizations (Fig. 6j).

In sum, this analysis highlights the value of mapping connections across both brain hemispheres and supports the utility of EM connectomics in both finding novel links between (previously considered distinct) pathways and identifying important distinctions within cell types known from light microscopy studies.

\section{Community organization}

Users are currently being recruited from Drosophila labs. We are starting with professional scientists, who are inherently incentivized for productivity and accuracy, because their own discoveries depend on their proofreading. Later on, we plan to expand recruitment to 
non-scientist volunteers. This is more complex, because "gamification" will be required to incentivize users (J. S. Kim et al. 2014).

During onboarding, users study self-guided training materials (see "Training Materials" on https://flywire.ai), and practice proofreading in a "Sandbox" dataset. Users are granted proofreading privileges in the real dataset after passing an entry test. In Wikipedia, unqualified or malicious users may introduce mistakes into articles. However, even without tests the completeness and accuracy of articles in Wikipedia tends to increase over time as users detect and correct omissions or errors in articles. Some studies have found that Wikipedia is approximately as accurate as traditional encyclopedias (Giles 2005). FlyWire utilizes the same basic mechanism of crowd wisdom as Wikipedia, iterative collaborative editing, while adding a safety layer through entry-level testing and subsequent spot checks of proofreading quality.

Members must consent to follow the FlyWire community principles (https://flywire.ai). As mentioned earlier, the most important principle is openness. Users may access information about any cell, such as cell type, proofreading status, or who made edits. Another important principle is fairness. Every edit to a neuron is regarded as a "micropublication," and the identity of the authoring member is recorded in the database along with the edit. Members agree to freely license their edits to others provided their contributions are attributed. When using FlyWire reconstructions in a scientific publication or presentation, users must obtain the agreement of other significant contributors, and make their neurons "public". Once "public" neurons are available to anyone (such as the neurons included in this publication). Such credit assignment procedures attempt to make FlyWire fair while maintaining its openness.

\section{DISCUSSION}

FlyWire is an implementation of our proposal for an open community to proofread an automated reconstruction of the entire Drosophila melanogaster brain. Interactive proofreading in FlyWire is enabled by a novel data structure for proofreading large connectomics datasets, the ChunkedGraph. As a demonstration of the segmentation and proofreading tools in FlyWire, we reconstructed 180 known mechanosensory neurons across both hemispheres and introduced new subdivisions of known cell types based on their connectivity and morphology. Further, we found new connections between the courtship song and escape pathways. Our results were corroborated by their replication in the other hemisphere. FlyWire's completeness of the brain allows researchers to identify all partners of a neuron. While we observed a striking hemispheric symmetry in the connectivity between the analyzed mechanosensory neurons, we also found asymmetric interactions of neurons crossing the midline, providing a potential mechanism for spatial localization of sounds, a challenging problem for flies with closely-spaced antennal auditory receivers (Morley et al. 2012).

It is likely that each whole brain connectome will require proofreading by many people for years, in spite of increases in the accuracy of automated reconstruction. We propose that whole-brain connectomics for each animal species could benefit from a decentralized approach that crowdsources proofreading to the researchers of that species. This approach would make 
circuits available with zero delay, accelerating research. Researchers would be able to prioritize proofreading of their own circuits of interest, and researchers could choose to proofread to any accuracy level required by their own scientific questions.

Using the current segmentation's mean backbone proofreading time of approximately 135 minutes per neuron, and an estimate that the Drosophila brain contains approximately 100,000 cells, a whole-brain connectome of these backbones with their existing twigs would require 112.5 person-years of proofreading assuming the use of automatic synapse detection. Ongoing improvements in both the automatic segmentation and the proofreading interface will reduce the number of errors further and make it possible to find and correct the remaining ones more rapidly. Proofreading can be sped up by having the computer automatically identify likely errors and suggest corrections (Zung et al. 2017).

At this writing, over 100 researchers from over 30 labs have been onboarded and trained for FlyWire, and membership is expanding. There are hundreds of labs studying Drosophila neural circuits worldwide, and the Drosophila research community has a long history of sharing and collaboration. Furthermore, the automated segmentation is now so accurate that interesting science can be discovered by only modest proofreading effort. 


\section{a}

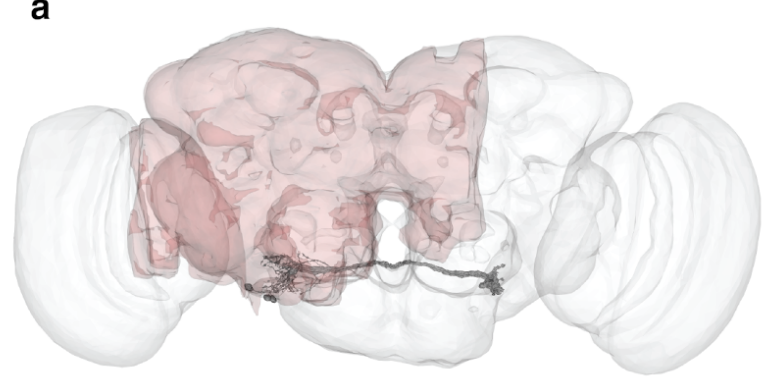

b

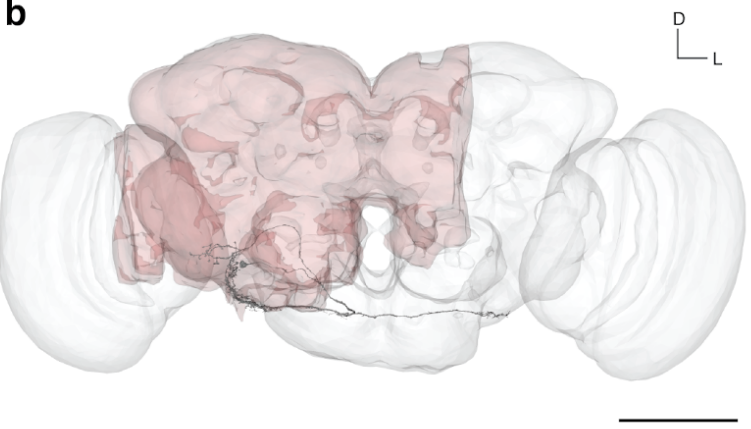

Extended Data Figure 1. Full brain rendering and comparison with the hemibrain. A neuropil rendering of the fly brain (white) is overlaid with a rendering of the hemibrain (see Methods) and proofread reconstructions of neurons from the antennal mechanosensory and motor center (AMMC). The proofread reconstructions of all (a) AMMC-B2 neurons from the right hemisphere and the (b) the AMMC-A2 neuron from the right hemisphere are added, illustrating how many neurons are only partially contained in the hemibrain dataset. Scale bar: $100 \mu \mathrm{m}$ 

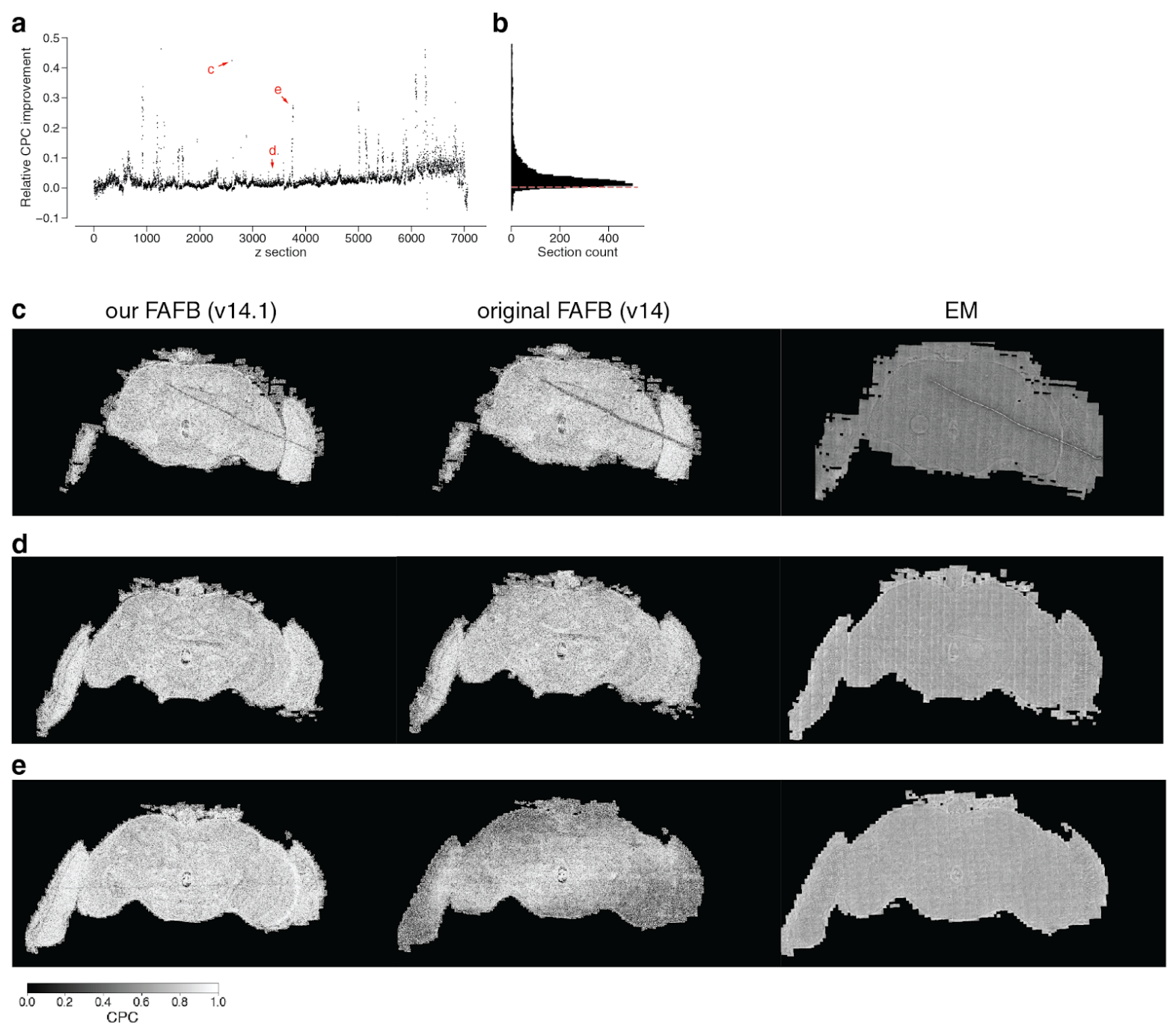

Extended Data Figure 2. Quality of EM image alignment. We calculated the chunked pearson correlation (CPC) between two neighboring sections in the original alignment (v14) and our re-aligned data (v14.1). (a) The relative change of CPC between the original and our re-aligned data per section (b) is almost always positive (dashed red line is at 0). (c, d, e) show examples from (a) where (c) the CPC improved through a better alignment around an artifact, (d) the CPC is almost identical and (e) the CPC overall improved due to a stretch of poorly aligned sections in the original data that were resolved in v14.1. 
a

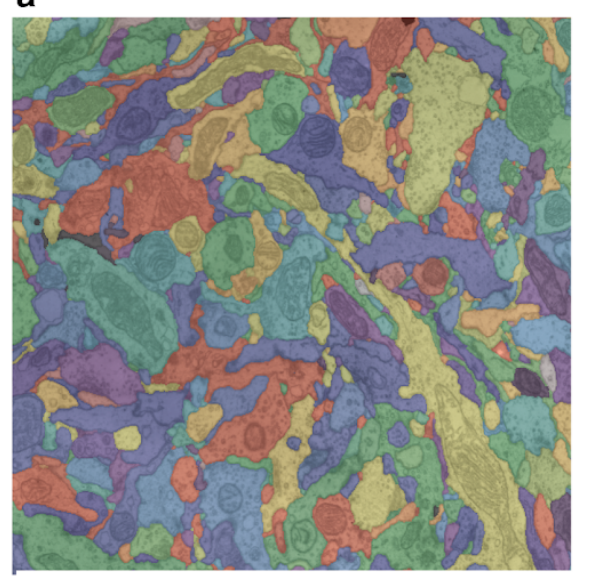

b

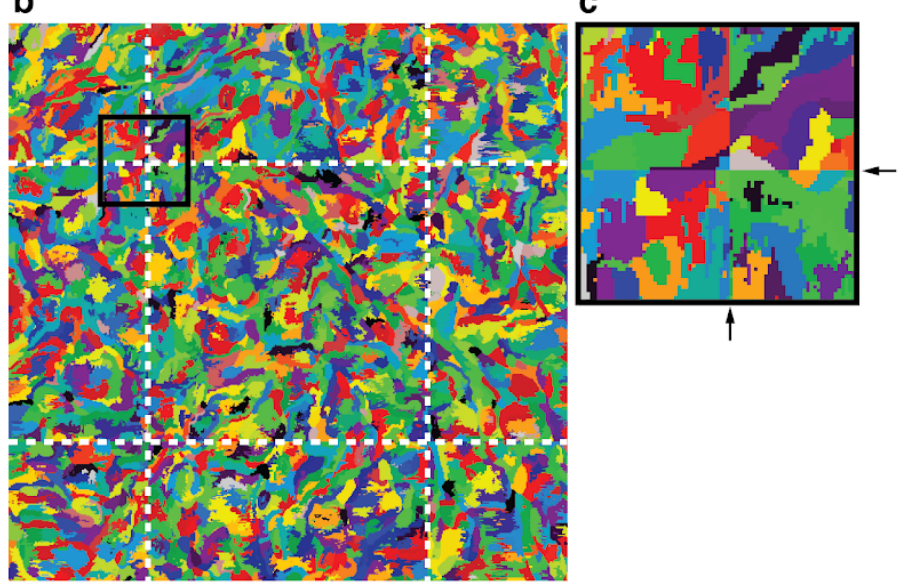

d
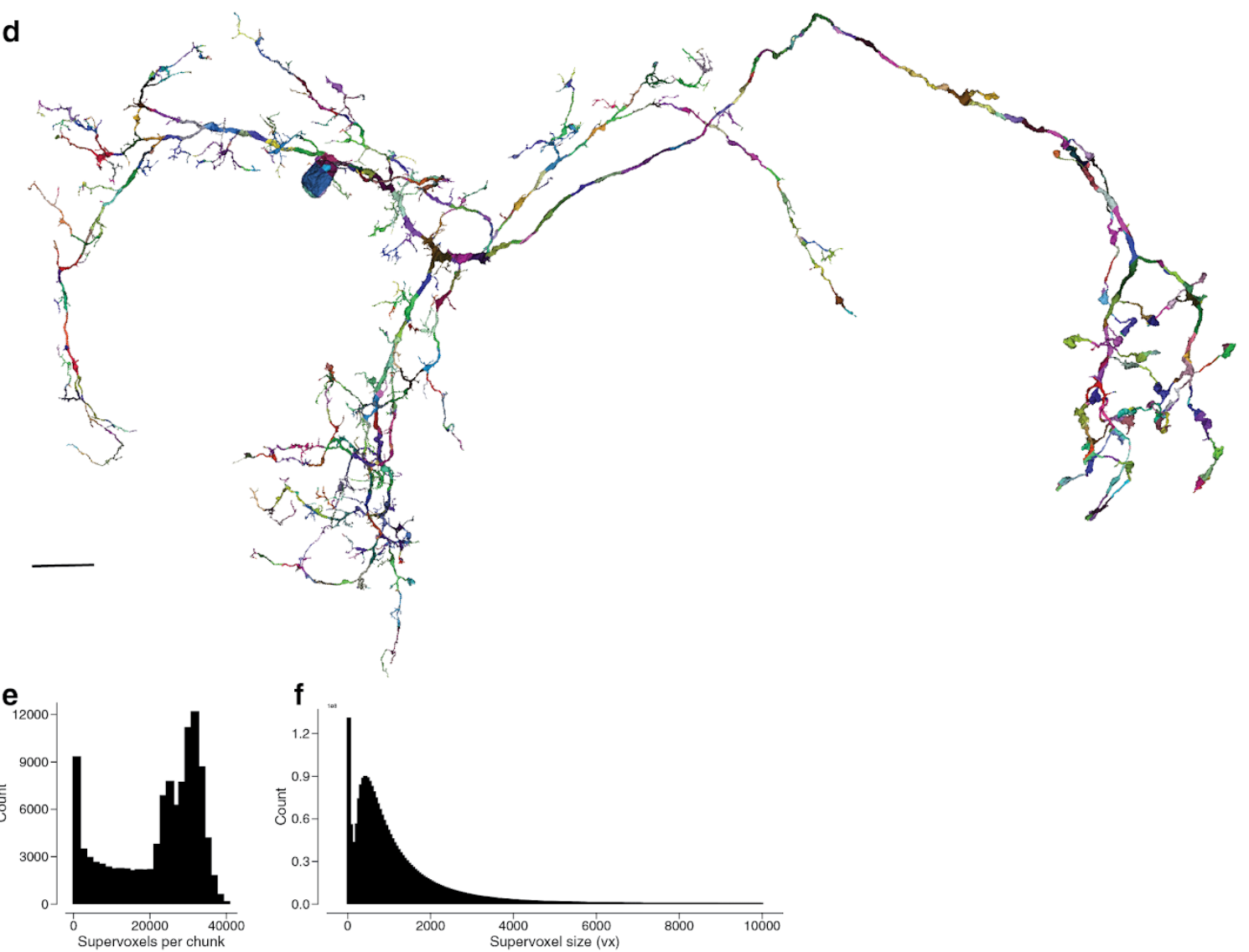

Extended Data Figure 3. Chunking the dataset. (a) Automated segmentation overlayed on the EM data. Each different color represents an individual putative neuron. (b) The underlying supervoxel data is chunked (white dotted lines) such that each supervoxel is fully contained in one chunk. (c) A close up view of the box in (b). (d) We apply the same chunking scheme to the meshes, requiring only minimal mesh recomputations after edits. (e) The irregular shape (not a box) of the fly brain leads to a high diversity of the number of supervoxels in each chunk (median: 25661). (f) The median supervoxel contains 792 voxels. All very small supervoxels ( $<200$ voxels) are the result of chunking. 


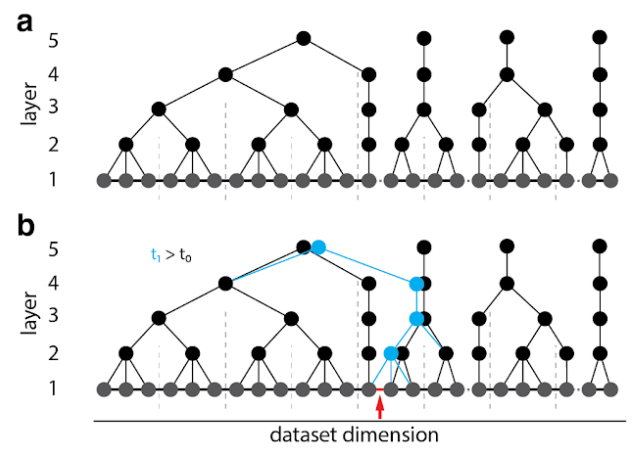

C

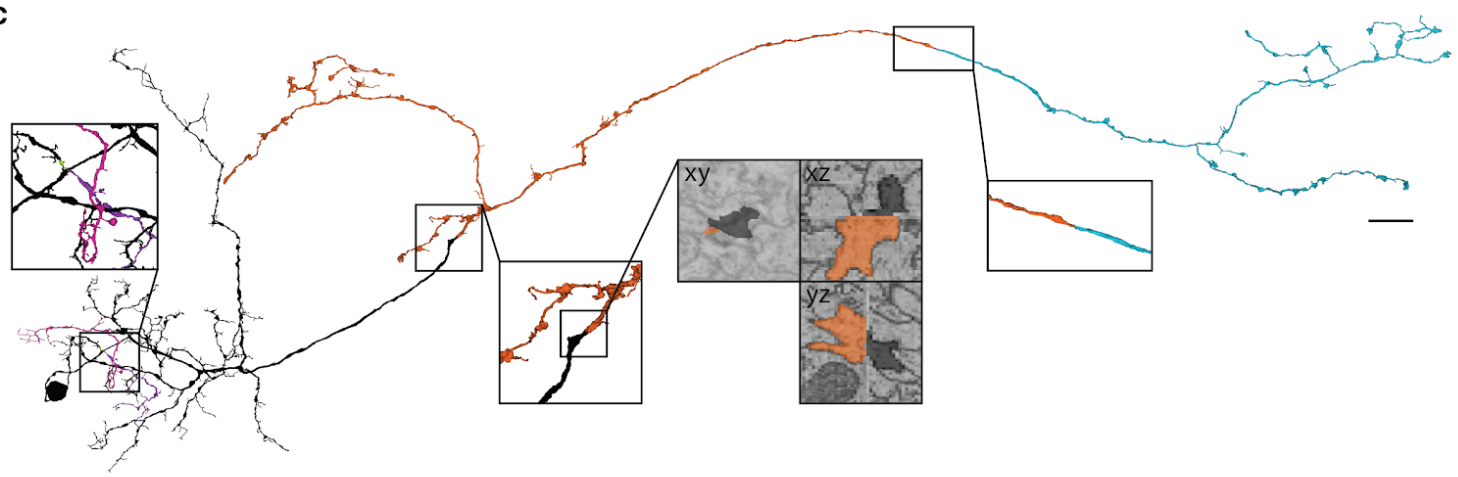

Extended Data Figure 4. Proofreading with the ChunkedGraph. (a, b) Analogous to the split operation in Fig. 3a, $b$ a merge operation does not require the loading and writing to and from the entire connected component since we can reuse the connected component information stored in the abstract nodes that did not see a change to their underlying graph. (c) During proofreading, all shown segments were added together. This is the same neuron for which removed segments were shown in Fig 2. Scale bar (c): $10 \mu \mathrm{m}$ 
bioRxiv preprint doi: https://doi.org/10.1101/2020.08.30.274225; this version posted August 30, 2020. The copyright holder for this preprint (which was not certified by peer review) is the author/funder, who has granted bioRxiv a license to display the preprint in perpetuity. It is made available under aCC-BY-ND 4.0 International license.

a

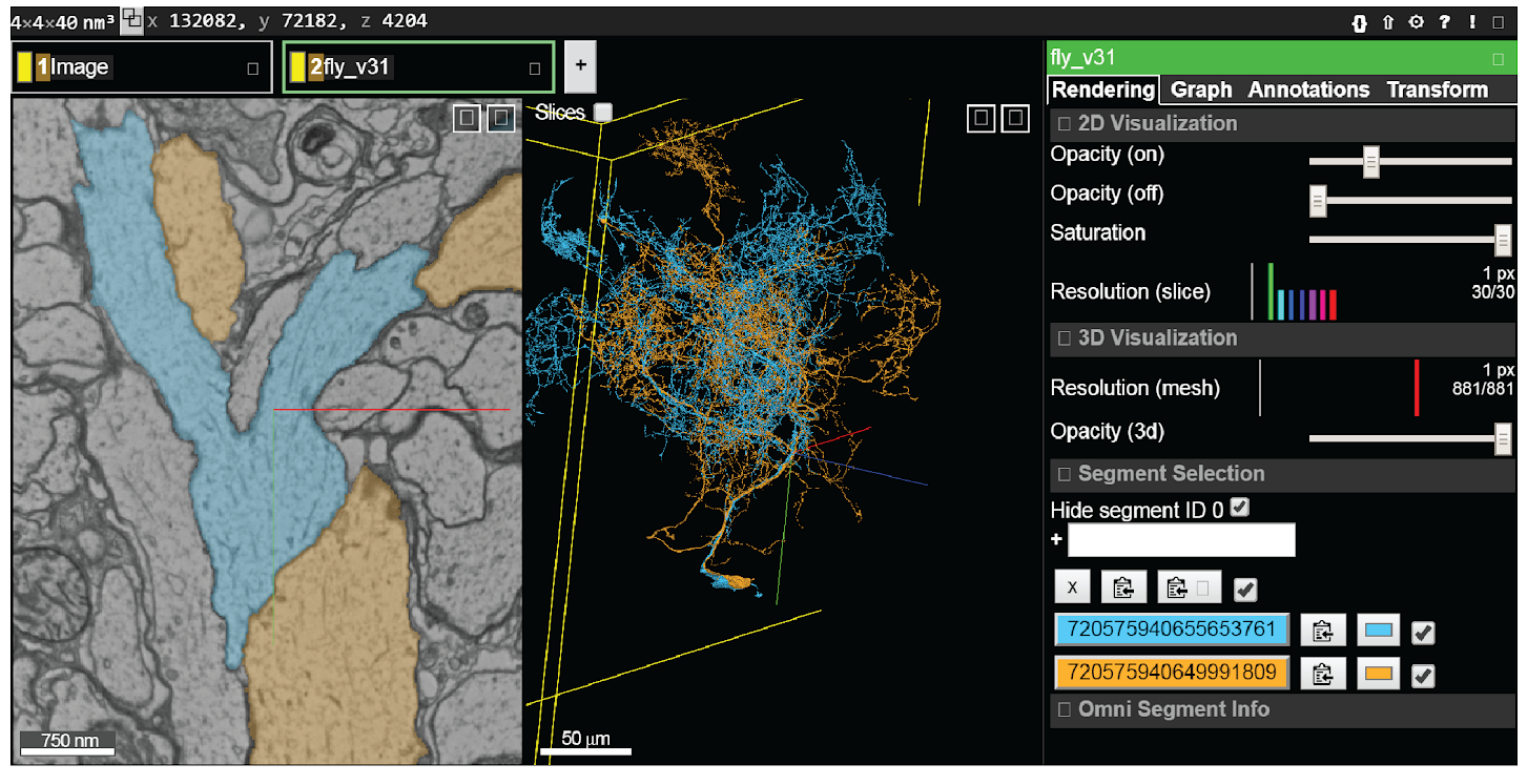

b

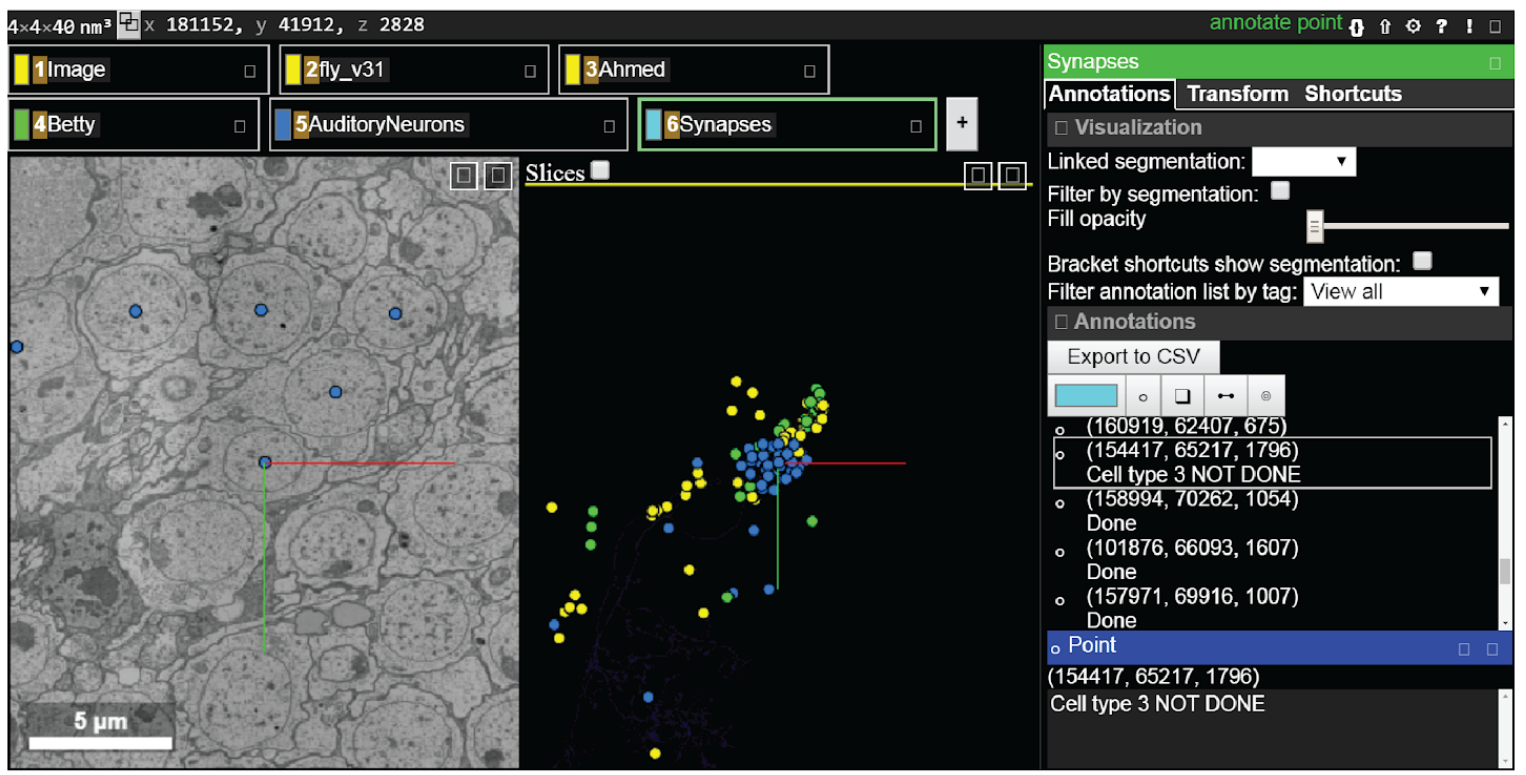

Extended Data Figure 5. The FlyWire proofreading platform. (a) The most common view in FlyWire displays the EM image in grayscale overlaid with segmentation in color (left panel), a 3D view of selected cell segments (middle), and menus with multiple proofreading tools (right). (b) Annotation tools include points, which can be used for a variety of purposes such as marking particular cells or synapses. 


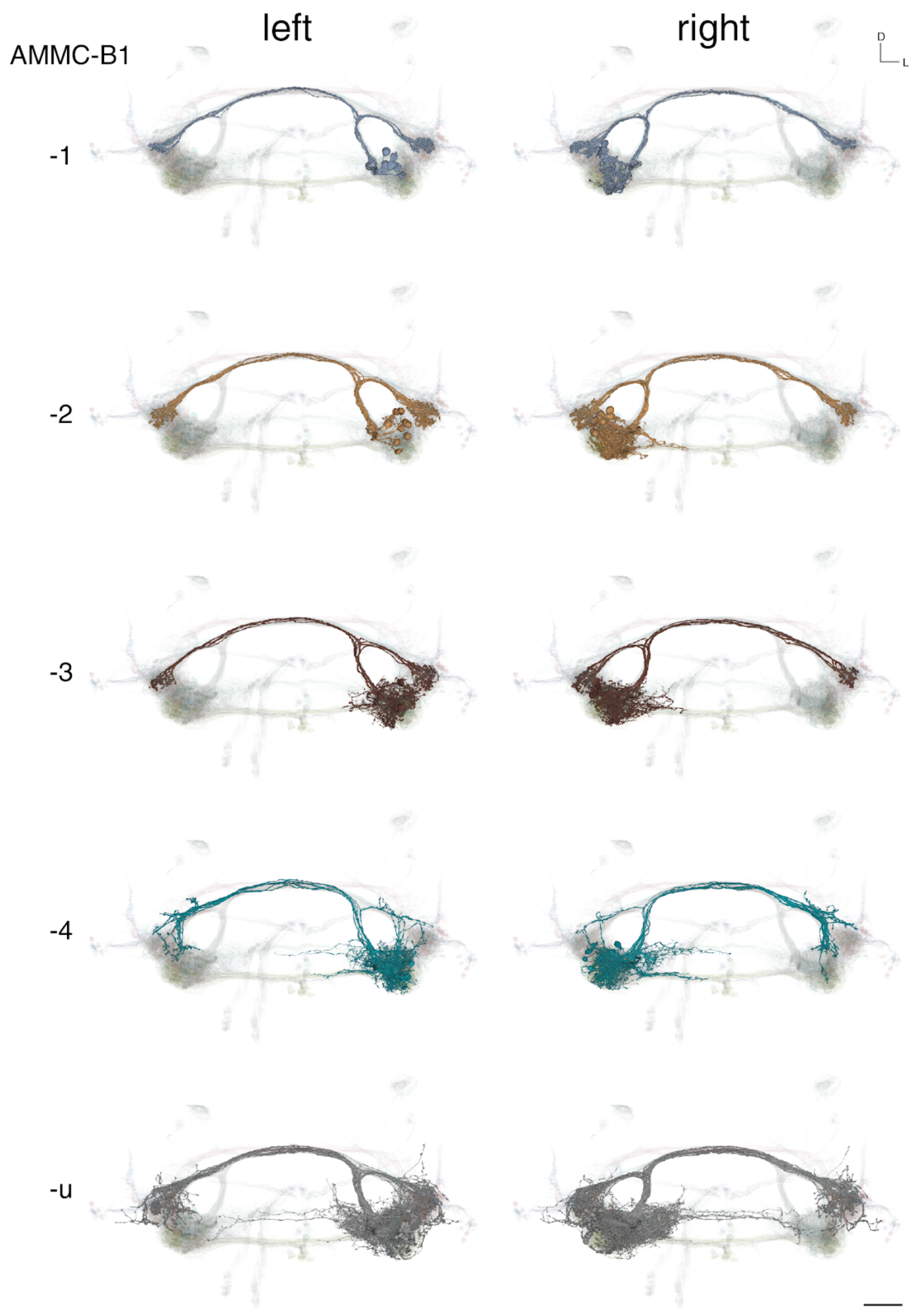

Extended Data Figure 6. Renderings of AMMC-B1 subtypes grouped by subtype and hemisphere. All 180 neurons included in the mechanosensory analysis are shown as backdrop. Scale bar: $25 \mu \mathrm{m}$ 
bioRxiv preprint doi: https://doi.org/10.1101/2020.08 30274225; this version posted August 30,2020 . The copyright holder for this preprint (which was not certified by peer review) is the author/funder, who has granted bioRxiv a license to display the preprint in perpetuity. It is made available under aCC-BY-ND 4.0 International license.

a

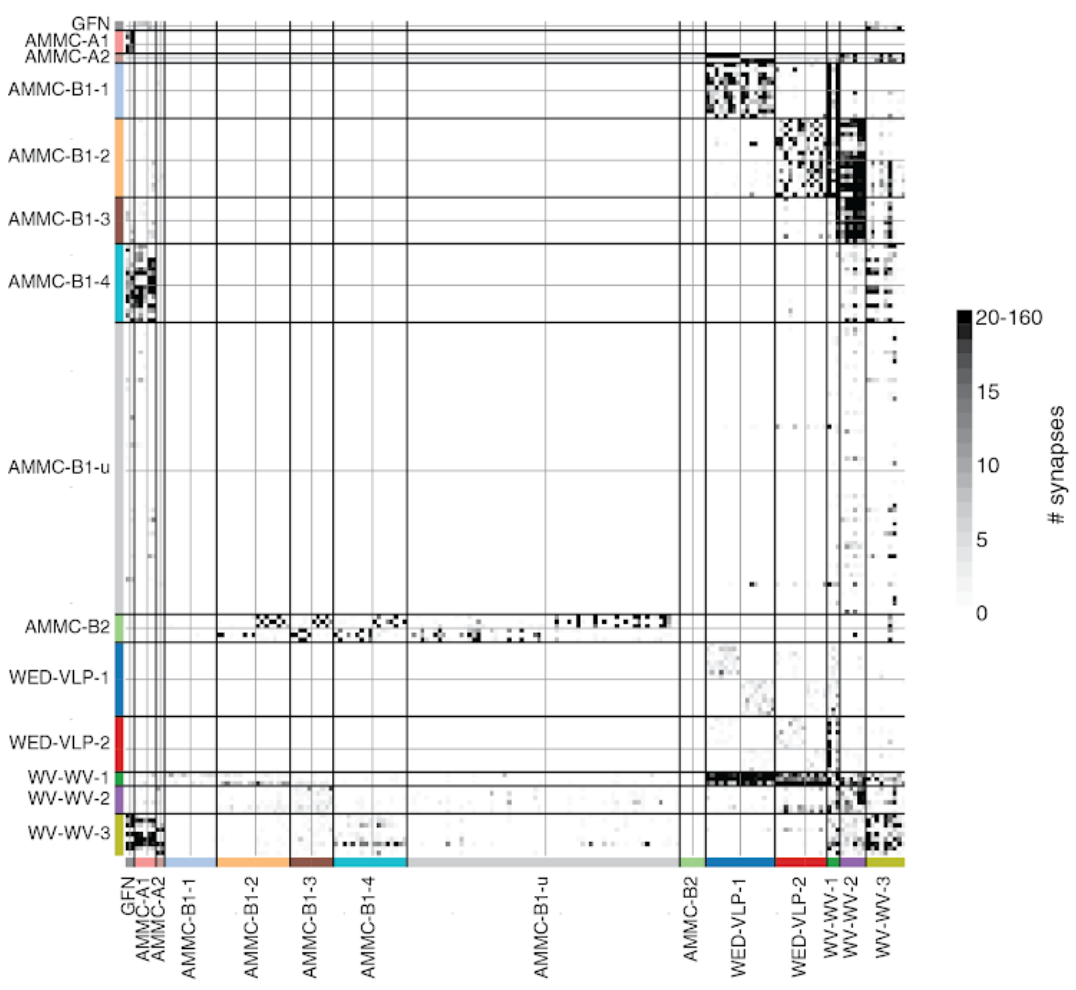

b

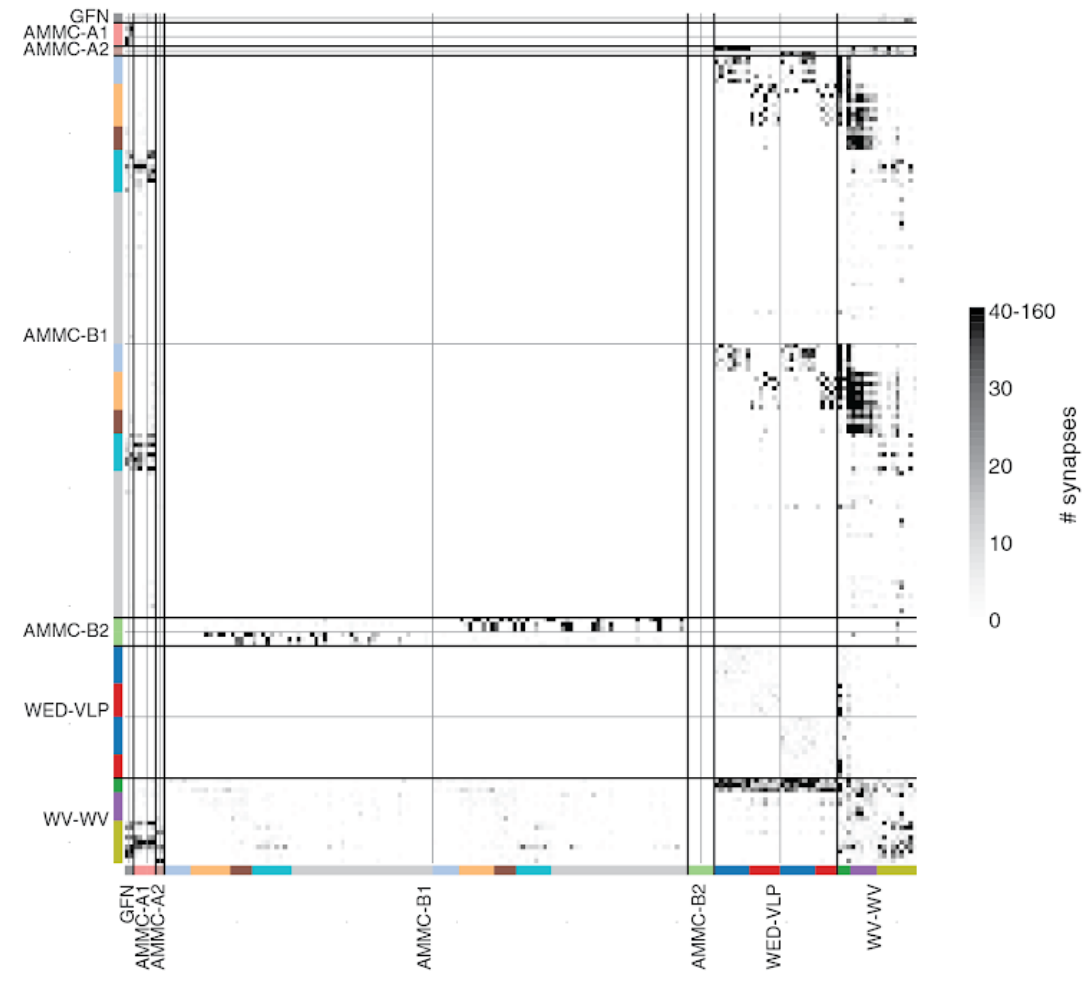

Extended Data Figure 7. Connectivity diagrams. (a) Diagram from Figure 6b reordered by subtype (b) Same diagram as in Figure $6 \mathrm{~b}$ with different colormap threshold. 


\begin{tabular}{|c|c|c|c|c|}
\hline Cell type & Subtype & Hemisphere & Count & Total count \\
\hline \multirow{2}{*}{ AMMC-A1 } & - & left & 3 & \multirow{2}{*}{5} \\
\hline & - & right & 2 & \\
\hline \multirow{2}{*}{ AMMC-A2 } & - & left & 1 & \multirow{2}{*}{2} \\
\hline & - & right & 1 & \\
\hline \multirow{10}{*}{ AMMC-B1 } & \multirow{2}{*}{1} & left & 6 & \multirow{10}{*}{119} \\
\hline & & right & 6 & \\
\hline & \multirow{2}{*}{2} & left & 9 & \\
\hline & & right & 8 & \\
\hline & \multirow{2}{*}{3} & left & 5 & \\
\hline & & right & 5 & \\
\hline & \multirow{2}{*}{4} & left & 9 & \\
\hline & & right & 8 & \\
\hline & \multirow{2}{*}{ u } & left & 32 & \\
\hline & & right & 31 & \\
\hline \multirow{2}{*}{ AMMC-B2 } & - & left & 3 & \multirow{2}{*}{6} \\
\hline & - & right & 3 & \\
\hline \multirow{2}{*}{ GFN } & - & left & 1 & \multirow{2}{*}{2} \\
\hline & - & right & 1 & \\
\hline \multirow{4}{*}{ WED-VLP } & \multirow{2}{*}{1} & left & 8 & \multirow{4}{*}{28} \\
\hline & & right & 8 & \\
\hline & \multirow{2}{*}{2} & left & 7 & \\
\hline & & right & 5 & \\
\hline \multirow{3}{*}{ WV-WV } & 1 & unpaired & 3 & \multirow{3}{*}{18} \\
\hline & 2 & unpaired & 6 & \\
\hline & 3 & unpaired & 9 & \\
\hline
\end{tabular}

Supplementary Table 1. Cell type counts for mechanosensory analysis 
bioRxiv preprint doi: https://doi.org/10.1101/2020.08.30.274225; this version posted August 30, 2020. The copyright holder for this preprint (which was not certified by peer review) is the author/funder, who has granted bioRxiv a license to display the preprint in perpetuity. It is made available under aCC-BY-ND 4.0 International license.

\begin{tabular}{|c|c|c|}
\hline Types & Link (colored) & Link (random colors) \\
\hline AMMC-A1, left & 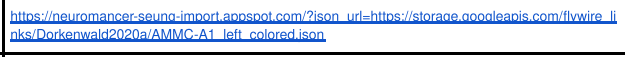 & 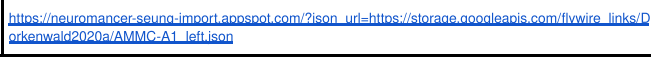 \\
\hline AMMC-A1, right & 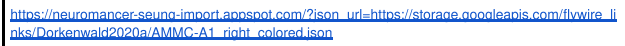 & 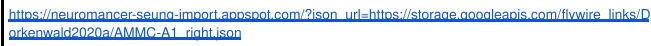 \\
\hline AMMC-A2, left & 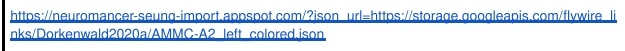 & 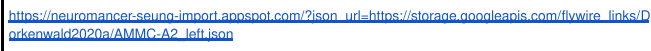 \\
\hline AMMC-A2, right & 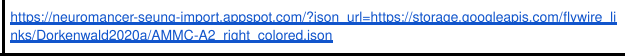 & $\begin{array}{l}\text { https://neuromancer-seuna-import.appspot.com/?ison url=https///storaqe.gooaleapis.com/filvwire links/D } \\
\text { orkenwald2020a/AMMC-A2 riaht.json }\end{array}$ \\
\hline AMMC-B1, left & 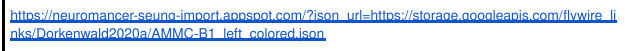 & 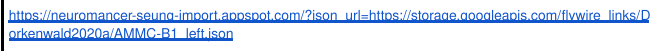 \\
\hline AMMC-B1, right & 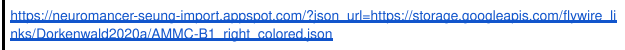 & 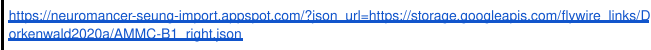 \\
\hline AMMC-B1-1, left & 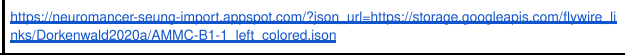 & $\begin{array}{l}\text { https://neuromancer-seuna-import. appspot.com/?ison url=https///storaqe.coooleapis.com/filvwire links/D } \\
\text { orkenwald2020a/AMMC-B1-1 left.jison }\end{array}$ \\
\hline AMMC-B1-1, right & 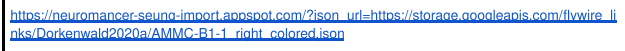 & 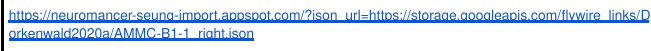 \\
\hline AMMC-B1-1/2/3, left & 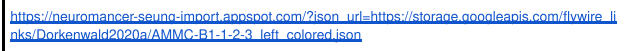 & 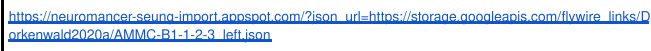 \\
\hline AMMC-B1-1/2/3, right & 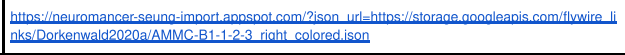 & $\begin{array}{l}\text { https://neuromancer-seuna-import.appspot.com/?ison url=https///storaqe.gooaleapis.com/flivwire links/D } \\
\text { orkenwald2020a/AMMC-B1-1-2-3 riaht.json }\end{array}$ \\
\hline AMMC-B1-2, left & 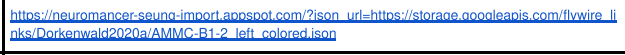 & 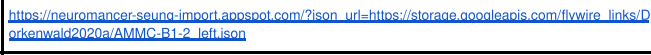 \\
\hline AMMC-B1-2, right & 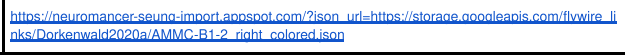 & 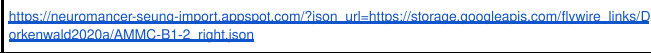 \\
\hline AMMC-B1-3, left & 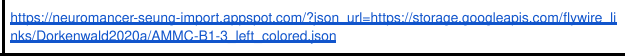 & 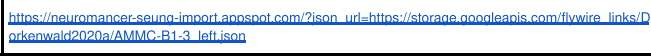 \\
\hline AMMC-B1-3, right & 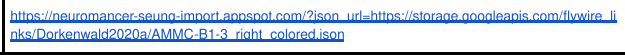 & 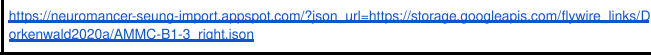 \\
\hline AMMC-B1-4, left & 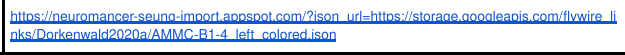 & $\begin{array}{l}\text { https:///neuromancer-seuna-import.appspot.com/?ison url=https///storaqe.,00oaleapis.com/filvwire links/D } \\
\text { arkenwald2020a/AMMC-B1-4 leftison }\end{array}$ \\
\hline AMMC-B1-4, right & 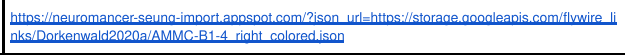 & 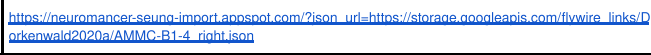 \\
\hline AMMC-B1-u, left & 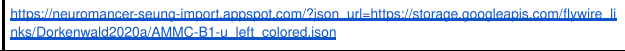 & $\begin{array}{l}\text { https://neuromancer-seuna-import.appspot.com/?ison url=https://storade.googleapis.com/fllwwire links/D } \\
\text { orkenwald2020a/AMMC-B1-u left.jison }\end{array}$ \\
\hline AMMC-B1-u, right & 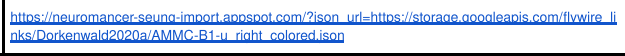 & 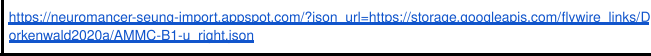 \\
\hline AMMC-B2, left & 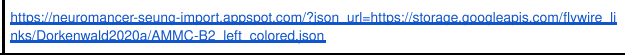 & 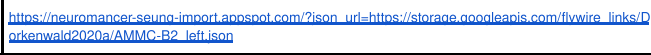 \\
\hline AMMC-B2, right & 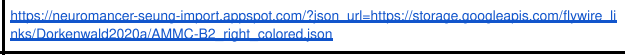 & 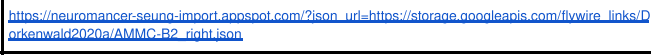 \\
\hline GFN, left & 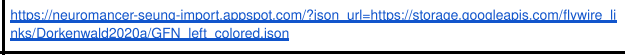 & 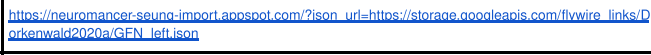 \\
\hline GFN, right & 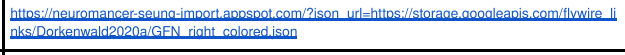 & 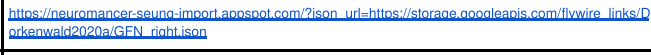 \\
\hline WED-VLP & 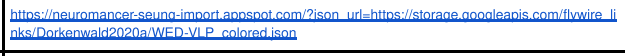 & 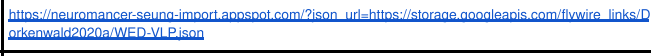 \\
\hline WED-VLP-1, left & 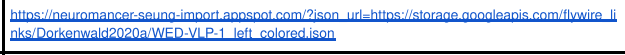 & 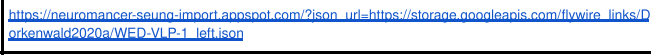 \\
\hline WED-VLP-1, right & 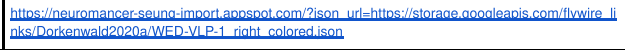 & 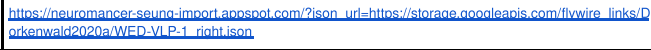 \\
\hline WED-VLP-2, left & 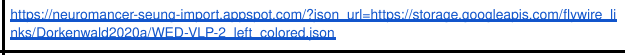 & 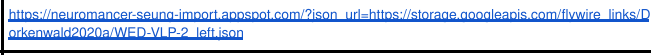 \\
\hline WED-VLP-2, right & 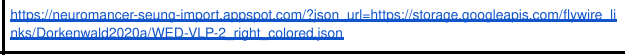 & 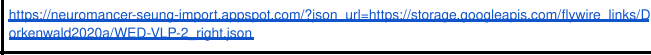 \\
\hline WV & 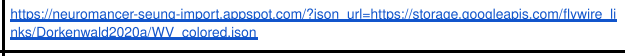 & 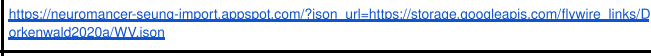 \\
\hline WV-WV-1 & 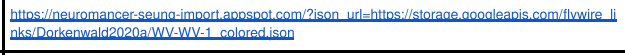 & 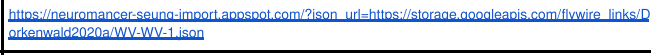 \\
\hline WV-WV-2 & 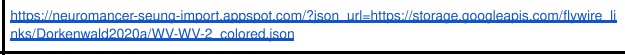 & 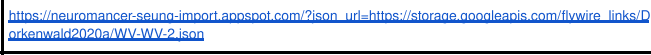 \\
\hline WV-WV-3 & 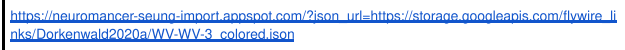 & 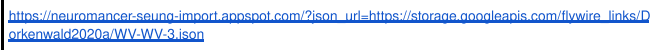 \\
\hline
\end{tabular}

Supplementary Table 2. Links to view neurons in Neuroglancer. Links forward users to our frontend and present neurons in 2D and 3D. No login is required to view these neurons; the selection of other neurons is, however, not possible without signing up to FlyWire and onboarding. Links are best viewed in a Chrome browser. 


\title{
METHODS
}

\begin{abstract}
Alignment
We started from the aligned dataset published by Zheng et al. (Zheng et al. 2018)(v14). Using the method outlined in Mitchell et al. (Mitchell et al. 2019) we trained neural networks through self-supervision to predict pairwise displacement fields between neighboring sections. Here, every location stores a vector pointing to its source location. We introduce a smoothness regularization into the training to ensure continuous transformations. This prior is relaxed at image artifacts such as cracks and folds. We first trained a convolutional neural network to detect image artifacts from a manually labeled training set, then used the predicted masks to adjust the smoothness prior during training of the displacement field network. We combine the pairwise displacement fields to generate a displacement field for every section, and apply the result to the data to create a newly aligned stack.
\end{abstract}

\section{Cross alignment registration and brain renderings}

Our alignment created a vector field for transformations from FlyWire's space (v14.1) to the original alignment space (v14). In order to transform data from v14 into v14.1 (e.g. synapses and brain renderings), we created an inverse transformation of the vector field at a resolution of $64 \mathrm{x}$ $64 \times 40 \mathrm{~nm}$. Locations in v14 are then transferred to v14.1 by applying the closest displacement vector from the inverse transformation.

The v14 brain rendering was acquired from the hemibrain website: https://flyconnectome.github.io/hemibrainr/reference/hemibrain.surf.html. The v14 whole brain neuropil rendering was acquired from the virtual fly brain website: https://fafb.catmaid.virtualflybrain.org/.

\section{Segmentation ground truth}

We made use of the publicly available ground truth from the CREMI challenge (https://cremi.org) to train our convolutional neural network for predicting affinities. We realigned these ground truth blocks as they contained misalignments as well.

\section{Segmentation}

We applied our segmentation pipeline as outlined in (Dorkenwald et al. 2019; Lee et al. 2017) without the use of long-range affinities. Additionally, we introduced a size-dependent threshold to break big, dumbbell shaped mergers occuring at low threshold. In the affinity graph, we ignored any edges between two large segments s1, s2 if mean(affinities(s1, s2)) < 0.5 and $\min (s 1, s 2)>$ 1,000 and $\max (s 1, s 2)>10,000$ representing supervoxel counts.

\section{Proofreading}

The ChunkedGraph is our backend database structure that enables parallel proofreading at scale. It stores the mean affinity graph of supervoxels and uses an octree to provide a spatial embedding for fast updates of the connected components representing neuronal segments. We adapted the Neuroglancer Frontend to command split and merge operations to our server 
backend. Every edit is stored with a timestamp allowing earlier states of the segmentation to be displayed at later times. To allow parallel proofreading of multiple users, we implement per-neuron locks where we process edits to the same neuron serially. Our server immediately starts remeshing of changed segments to give the user 3D feedback. Remeshing takes seconds to tens of seconds.

\section{ChunkedGraph performance analysis}

During the beta phase of FlyWire, we measured server response times for various requests by all users (Fig. 3e,f). These numbers reflect real interactions and are affected by server and database load and are therefore an underestimate of the capability of our system.

We used real split edits as the basis for the comparison of the ChunkedGraph with a naive implementation that had been performed in FlyWire's beta phase prior to this analysis. For this comparison, we used the same BigTable table but ignored the additional ChunkedGraph hierarchy for the naive implementation.

\section{Proofreading evaluation}

183 neurons were proofread by 13 proofreaders consisting of both scientists and expert tracers from the Seung and Murthy labs. For 49 of these we measured the time it took the neuron in the first round. For all 183, we only proofread major components (backbones) and did not attempt to look for orphan twigs, small microtubule-free terminal branches, as defined in (Schneider-Mizell et al. 2016). However, proofreaders still added those that were immediately obvious to them.

To obtain the number of edits for each neuron, we excluded edits made to chop neurons apart for inspection, that were later reversed by merging those pieces back together. We also excluded edits to a segment that was removed from that neuron later in the proofreading process. Consider the example where a neuron was merged to a big component containing other neuronal segments. We did not count edits for removing other neuronal segments from that component towards the edit count for the neuron at hand. More specifically, we only considered merge operations where all merge locations remained in the neuron at the end of proofreading and split operations where exactly one side of the split was contained in the final neuron.

We calculated the volumetric change of edits and the volumetric completeness from the segmentation by collecting the supervoxels that were added/removed and adding up the voxel within each of them. We then multiplied this number with the nominal resolution of the segmentation (16 × $16 \times 40 \mathrm{~nm})$.

\section{Twig and backbone synapse evaluation}

We randomly collected 999 synapses from all synapses predicted by Buhmann et al. (Buhmann et al. 2019). One expert evaluated all synapses as true positive (615), false positive synapses (285) or ambiguous (99). Next, this expert evaluated the reconstructions of the pre- and postsynaptic sides of the true positive synapses as either belonging to a twig that was attached 
to a backbone ("twig - attached"), twig that was not attached to a backbone ("twig - orphan") or "backbone."

\section{Identifying all cells within a class}

We aimed to find every cell of the mechanosensory types investigated here. To do so, a location was chosen in the soma tract of a cell lineage, where proximal neurites were tightly grouped into a clear bundle, often surrounded by glia. Alternatively, in some cell types without tightly clustered proximal neurites, a location was chosen in a distinctive region of the backbone where these cells showed bundling. By examining in $X Y, Y Z$ and $X Z$, a view was chosen that displayed the bundle in cross-section, to ensure that all cells in the bundle were visible. Every neuron in that cross-section was then examined to find the desired cell type. Any neuron that could not be classified was proofread until identification was possible. We expect this approach to reveal most or all cells within a lineage, however there could be reasons why some might be missed (such as a proximal neurite that travels outside the bundle). Locations used: AMMC-A1, right: (103406, 54035, 4640), left: (159748, 56141, 3678). AMMC-B2 commissure: (132104, 71166, 3416). WED-VLP, left: (172786, 69380, 2254), right: (88476, 65205, 3043). WED-WED and AMMC-AMMC (same midline soma tract): (132008, 84118, 4272). AMMC-B1: not all were tightly bundled, used two locations per hemisphere for cross-sections: left: $(151298,69205$, 1686) and (152447, 61490, 3218), right: $(111828,67177,2127)$ and $(111214,60441,3615)$. GF and AMMC-A2: only one cell exists per hemisphere (for AMMC-A2, commissure was examined for confirmation).

\section{Synapse proofreading}

We used automatically detected synapses by Buhmann et al. (Buhmann et al. 2019) for the analysis of the mechanosensory connectome (Fig. 6). During analysis, we noticed a higher occurrence of false positive synapses between some cell types. These were usually cell types that had a high number of contacts due to spatial proximity. We randomly inspected about 25 synapses per <cell type > to <cell type> (eg. AMMC-B1 to WED-VLP or AMMC-B1 to AMMC-B1) and disregarded connections with mostly false positive or questionable synapses. This exclusion mostly affected connections within cell types (eg. AMMC-B1 to AMMC-B1). We did not remove single false positive synapses and the remaining <cell type > to <cell type > connections reported in Figure 6 still have false positive synapses among them.

\section{Celltype division}

We divided cell-types into sub-types according to their connectivity and then verified the subdivision morphologically. We list all numbers in Supplementary Table 1.

WED-VLP: Neurons receiving more than 10 synapses from the ipsilateral AMMC-A2 were classified as WED-VLP-1, all others as WED-VLP-2.

$A M M C-B 1$ : We first selected neurons with more than 50 synapses onto any WED-VLP. These were then labeled as AMMC-B1-1 if they made more than $50 \%$ of their WED-VLP synapses onto WED-VLP-1 and AMMC-B1-2 otherwise. Out of the remaining AMMC-B1 neurons (not -1 or -2), those with more than 80 synapses onto any WV-WV neuron were labeled as AMMC-B1-3. From the remaining $A M M C-B 1$ cells, we labeled those as AMMC-B1-4 if they made at least 20 
synapses onto AMMC-A1, AMMC-A2 and GFN cells combined. The remaining cells were classified as AMMC-B1-u.

$W V-W V$ : First, we labeled all WV-WV neurons with more than 20 synapses onto AMMC-A1, AMMC-A2 and GFN combined as WV-WV-3. Out of the remaining neurons, we labeled those with more than 100 synapses onto WED-VLP as WV-WV-1. WV-WV-2 was made up of all remaining WV-WV neurons. 


\section{ACKNOWLEDGMENTS}

HSS and MM acknowledge support from NIH BRAIN Initiative RF1 MH117815-01 to HSS and MM. HSS further acknowledges support from the Mathers Foundation, as well as assistance from Google and Amazon.

We are grateful for support with FAFB imagery by Stephan Saalfeld, Eric Trautman, and Davi Bock. We thank Greg Jefferis, Davi Bock, and Albert Cardona for advice regarding the community. We thank Greg Jefferis and Philipp Schlegel with help with the brain renderings. We thank Garrett McGrath for computer system administration, and May Husseini for project administration. We are grateful to J. Maitin-Shepard for neuroglancer. We are grateful to J. Buhmann and J. Funke for discussions and for making their automatically predicted synapses publicly available. We thank Nuno da Costa and Agnes Bodor for feedback on the proofreading system during its development. We thank the Allen Institute for Brain Science founder, Paul G. Allen, for his vision, encouragement and support.

This work was supported by the Intelligence Advanced Research Projects Activity (IARPA) via Department of Interior/ Interior Business Center (Dol/IBC) contract numbers D16PC00003, D16PC00004, and D16PC0005. The U.S. Government is authorized to reproduce and distribute reprints for Governmental purposes notwithstanding any copyright annotation thereon.

Disclaimer: The views and conclusions contained herein are those of the authors and should not be interpreted as necessarily representing the official policies or endorsements, either expressed or implied, of IARPA, Dol/IBC, or the U.S. Government.

\section{CONTRIBUTIONS}

TM, NK realigned the dataset with methods developed by EM, BN, TM and infrastructure by SP, $\mathrm{ZJ}, \mathrm{JAB}, \mathrm{SM}$ wrote code for masking defects and misalignments. $\mathrm{KL}$ trained the convolutional net for boundary detection, using ground truth data realigned by DI. JW used the convolutional net to generate an affinity map which was segmented by RL. SD, NK created the proofreading system with input from JZ and ZA. NK, MAC, OO and AH adapted and improved Neuroglancer for proofreading and annotations. SD, FC, CSM, CSJ, DB built the server infrastructure to host FlyWire and manage users. WMS ingested the images into cloud storage. CM managed the community, designed the training tutorials and trained proofreaders. $C B, J G, D D, L E R, S K, A B$, $\mathrm{JH}, \mathrm{MM}, \mathrm{SM}, \mathrm{BS}, \mathrm{KW}, \mathrm{RW}$ tested the site and proofread neurons. CM, JG devised neuron annotation procedures. SCY managed proofreaders and evaluated twigs. SD evaluated the proofreading system. SD, CM analyzed the data. SD, CM, HSS, MM wrote the manuscript. HSS, MM led the effort. 


\section{REFERENCES}

Ahrens, Misha B., Michael B. Orger, Drew N. Robson, Jennifer M. Li, and Philipp J. Keller. 2013. "Whole-Brain Functional Imaging at Cellular Resolution Using Light-Sheet Microscopy." Nature Methods 10 (5): 413-20.

Allen, Marcus J., Tanja A. Godenschwege, Mark A. Tanouye, and Pauline Phelan. 2006. "Making an Escape: Development and Function of the Drosophila Giant Fibre System." Seminars in Cell \& Developmental Biology 17 (1): 31-41.

Azevedo, Anthony W., and Rachel I. Wilson. 2017. "Active Mechanisms of Vibration Encoding and Frequency Filtering in Central Mechanosensory Neurons." Neuron 96 (2): 446-60.e9.

Buhmann, J., A. Sheridan, S. Gerhard, and R. Krause. 2019. "Automatic Detection of Synaptic Partners in a Whole-Brain Drosophila EM Dataset." bioRxiv. https://www.biorxiv.org/content/10.1101/2019.12.12.874172v1.abstract.

Buhmann, Julia, Renate Krause, Rodrigo Ceballos Lentini, Nils Eckstein, Matthew Cook, Srinivas Turaga, and Jan Funke. 2018. "Synaptic Partner Prediction from Point Annotations in Insect Brains." In Medical Image Computing and Computer Assisted Intervention - MICCAI 2018, 309-16. Springer International Publishing.

Chang, Fay, Jeffrey Dean, Sanjay Ghemawat, Wilson C. Hsieh, Deborah A. Wallach, Mike Burrows, Tushar Chandra, Andrew Fikes, and Robert E. Gruber. 2008. "Bigtable: A Distributed Storage System for Structured Data." ACM Transactions on Computer Systems, 4, 26 (2): 1-26.

Clemens, Jan, Cyrille C. Girardin, Pip Coen, Xiao-Juan Guan, Barry J. Dickson, and Mala Murthy. 2015. "Connecting Neural Codes with Behavior in the Auditory System of Drosophila." Neuron 87 (6): 1332-43.

Coen, Philip, Jan Clemens, Andrew J. Weinstein, Diego A. Pacheco, Yi Deng, and Mala Murthy. 2014. "Dynamic Sensory Cues Shape Song Structure in Drosophila." Nature 507 (7491): 233-37.

Cook, Steven J., Travis A. Jarrell, Christopher A. Brittin, Yi Wang, Adam E. Bloniarz, Maksim A. Yakovlev, Ken C. Q. Nguyen, et al. 2019. "Whole-Animal Connectomes of Both Caenorhabditis Elegans Sexes." Nature 571 (7763): 63-71.

DasGupta, Shamik, Clara Howcroft Ferreira, and Gero Miesenböck. 2014. "FoxP Influences the Speed and Accuracy of a Perceptual Decision in Drosophila." Science 344 (6186): 901-4.

Deutsch, D., D. A. Pacheco, and L. J. Encarnacion-Rivera. 2020. "The Neural Basis for a Persistent Internal State in Drosophila Females." bioRxiv. https://www.biorxiv.org/content/10.1101/2020.02.13.947952v1.abstract.

Dolan, Michael-John, Ghislain Belliart-Guérin, Alexander Shakeel Bates, Shahar Frechter, Aurélie Lampin-Saint-Amaux, Yoshinori Aso, Ruairí J. V. Roberts, et al. 2018. "Communication from Learned to Innate Olfactory Processing Centers Is Required for Memory Retrieval in Drosophila." Neuron 100 (3): 651-68.e8.

Dorkenwald, S., N. L. Turner, T. Macrina, K. Lee, and R. Lu. 2019. "Binary and Analog Variation of Synapses between Cortical Pyramidal Neurons." bioRxiv. https://www.biorxiv.org/content/10.1101/2019.12.29.890319v1.abstract.

Dorkenwald, Sven, Philipp J. Schubert, Marius F. Killinger, Gregor Urban, Shawn Mikula, Fabian Svara, and Joergen Kornfeld. 2017. "Automated Synaptic Connectivity Inference for Volume Electron Microscopy." Nature Methods, February. https://doi.org/10.1038/nmeth.4206.

Duistermars, Brian J., Barret D. Pfeiffer, Eric D. Hoopfer, and David J. Anderson. 2018. "A Brain Module for Scalable Control of Complex, Multi-Motor Threat Displays." Neuron 100 (6): 1474-90.e4.

Felsenberg, Johannes, Pedro F. Jacob, Thomas Walker, Oliver Barnstedt, Amelia J. Edmondson-Stait, Markus W. Pleijzier, Nils Otto, et al. 2018. "Integration of Parallel Opposing Memories Underlies Memory Extinction." Cell 175 (3): 709-22.e15.

Giles, Jim. 2005. "Internet Encyclopaedias Go Head to Head." Nature 438 (7070): 900-901.

Haehn, D., S. Knowles-Barley, M. Roberts, J. Beyer, N. Kasthuri, J. W. Lichtman, and H. Pfister. 2014. "Design and Evaluation of Interactive Proofreading Tools for Connectomics." IEEE Transactions on Visualization and Computer Graphics 20 (12): 2466-75.

Heinrich, Larissa, Jan Funke, Constantin Pape, Juan Nunez-Iglesias, and Stephan Saalfeld. 2018. 
"Synaptic Cleft Segmentation in Non-Isotropic Volume Electron Microscopy of the Complete Drosophila Brain." In Medical Image Computing and Computer Assisted Intervention - MICCAI 2018, 317-25. Springer International Publishing.

Huang, Gary B., Louis K. Scheffer, and Stephen M. Plaza. 2018. "Fully-Automatic Synapse Prediction and Validation on a Large Data Set." Frontiers in Neural Circuits 12 (October): 87.

Jain, Viren, H. Sebastian Seung, and Srinivas C. Turaga. 2010. "Machines That Learn to Segment Images: A Crucial Technology for Connectomics." Current Opinion in Neurobiology. https://doi.org/10.1016/j.conb.2010.07.004.

Januszewski, Michał, Jörgen Kornfeld, Peter H. Li, Art Pope, Tim Blakely, Larry Lindsey, Jeremy Maitin-Shepard, Mike Tyka, Winfried Denk, and Viren Jain. 2018. "High-Precision Automated Reconstruction of Neurons with Flood-Filling Networks." Nature Methods 15 (8): 605-10.

Kamikouchi, Azusa, Hidehiko K. Inagaki, Thomas Effertz, Oliver Hendrich, André Fiala, Martin C. Göpfert, and Kei Ito. 2009. "The Neural Basis of Drosophila Gravity-Sensing and Hearing." Nature 458 (7235): 165-71.

Kim, H., M. Horigome, Y. Ishikawa, and F. Li. 2020. "Wiring Patterns from Auditory Sensory Neurons to the Escape and Song-relay Pathways in Fruit Flies." Journal of. https://onlinelibrary.wiley.com/doi/abs/10.1002/cne.24877.

Kim, Jinseop S., Matthew J. Greene, Aleksandar Zlateski, Kisuk Lee, Mark Richardson, Srinivas C. Turaga, Michael Purcaro, et al. 2014. "Space-Time Wiring Specificity Supports Direction Selectivity in the Retina." Nature 509 (7500): 331-36.

Knowles-Barley, Seymour, Verena Kaynig, Thouis Ray Jones, Alyssa Wilson, Joshua Morgan, Dongil Lee, Daniel Berger, Narayanan Kasthuri, Jeff W. Lichtman, and Hanspeter Pfister. 2016. "RhoanaNet Pipeline: Dense Automatic Neural Annotation." arXiv [q-bio.NC]. arXiv. http://arxiv.org/abs/1611.06973.

Kreshuk, Anna, Jan Funke, Albert Cardona, and Fred A. Hamprecht. 2015. "Who Is Talking to Whom: Synaptic Partner Detection in Anisotropic Volumes of Insect Brain." In Medical Image Computing and Computer-Assisted Intervention -- MICCAI 2015, 661-68. Springer International Publishing.

Lai, Jason Sih-Yu, Shih-Jie Lo, Barry J. Dickson, and Ann-Shyn Chiang. 2012. "Auditory Circuit in the Drosophila Brain." Proceedings of the National Academy of Sciences of the United States of America 109 (7): 2607-12.

Lee, Kisuk, Nicholas Turner, Thomas Macrina, Jingpeng Wu, Ran Lu, and H. Sebastian Seung. 2019. "Convolutional Nets for Reconstructing Neural Circuits from Brain Images Acquired by Serial Section Electron Microscopy." Current Opinion in Neurobiology. https://doi.org/10.1016/j.conb.2019.04.001.

Lee, Kisuk, Jonathan Zung, Peter Li, Viren Jain, and H. Sebastian Seung. 2017. "Superhuman Accuracy on the SNEMI3D Connectomics Challenge." arXiv [cs.CV], May. https://arxiv.org/abs/1706.00120.

Li, Peter H., Larry F. Lindsey, Michał Januszewski, Zhihao Zheng, Alexander Shakeel Bates, István Taisz, Mike Tyka, et al. 2019. "Automated Reconstruction of a Serial-Section EM Drosophila Brain with Flood-Filling Networks and Local Realignment." bioRxiv. https://doi.org/10.1101/605634.

Maitin-Shepard, J. 2020. "Neuroglancer." 2020. https://github.com/google/neuroglancer.

Meinertzhagen, Ian A. 2018. "Of What Use Is Connectomics? A Personal Perspective on the Drosophila Connectome." The Journal of Experimental Biology 221 (10). https://doi.org/10.1242/jeb.164954.

Mitchell, Eric, Stefan Keselj, Sergiy Popovych, Davit Buniatyan, and H. Sebastian Seung. 2019. "Siamese Encoding and Alignment by Multiscale Learning with Self-Supervision." arXiv [cs.CV]. arXiv. http://arxiv.org/abs/1904.02643.

Morley, Erica L., Thomas Steinmann, Jérôme Casas, and Daniel Robert. 2012. "Directional Cues in Drosophila Melanogaster Audition: Structure of Acoustic Flow and Inter-Antennal Velocity Differences." The Journal of Experimental Biology 215 (Pt 14): 2405-13.

Owald, David, Johannes Felsenberg, Clifford B. Talbot, Gaurav Das, Emmanuel Perisse, Wolf Huetteroth, and Scott Waddell. 2015. "Activity of Defined Mushroom Body Output Neurons Underlies Learned Olfactory Behavior in Drosophila." Neuron 86 (2): 417-27.

Pézier, Adeline P., Sami H. Jezzini, Jonathan P. Bacon, and Jonathan M. Blagburn. 2016. "Shaking B Mediates Synaptic Coupling between Auditory Sensory Neurons and the Giant Fiber of Drosophila Melanogaster." PloS One 11 (4): e0152211.

Phelan, Pauline, L. Ann Goulding, Jennifer L. Y. Tam, Marcus J. Allen, Rebecca J. Dawber, Jane A. 
Davies, and Jonathan P. Bacon. 2008. "Molecular Mechanism of Rectification at Identified Electrical Synapses in the Drosophila Giant Fiber System." Current Biology: CB 18 (24): 1955-60.

Priedhorsky, Reid, Jilin Chen, Shyong (tony) K. Lam, Katherine Panciera, Loren Terveen, and John Riedl. 2007. "Creating, Destroying, and Restoring Value in Wikipedia." In Proceedings of the 2007 International ACM Conference on Supporting Group Work, 259-68.

Reyn, Catherine R. von, Patrick Breads, Martin Y. Peek, Grace Zhiyu Zheng, W. Ryan Williamson, Alyson L. Yee, Anthony Leonardo, and Gwyneth M. Card. 2014. "A Spike-Timing Mechanism for Action Selection." Nature Neuroscience 17 (7): 962-70.

Schneider-Mizell, Casey M., Stephan Gerhard, Mark Longair, Tom Kazimiers, Feng Li, Maarten F. Zwart, Andrew Champion, et al. 2016. "Quantitative Neuroanatomy for Connectomics in Drosophila." eLife 5 (March). https://doi.org/10.7554/eLife.12059.

Seelig, Johannes D., and Vivek Jayaraman. 2015. "Neural Dynamics for Landmark Orientation and Angular Path Integration." Nature 521 (7551): 186-91.

Staffler, Benedikt, Manuel Berning, Kevin M. Boergens, Anjali Gour, Patrick van der Smagt, and Moritz Helmstaedter. 2017. "SynEM, Automated Synapse Detection for Connectomics." eLife 6 (July). https://doi.org/10.7554/eLife.26414.

Takemura, Shin-Ya, Arjun Bharioke, Zhiyuan Lu, Aljoscha Nern, Shiv Vitaladevuni, Patricia K. Rivlin, William T. Katz, et al. 2013. "A Visual Motion Detection Circuit Suggested by Drosophila Connectomics." Nature 500 (7461): 175-81.

Tootoonian, Sina, Philip Coen, Risa Kawai, and Mala Murthy. 2012. "Neural Representations of Courtship Song in the Drosophila Brain." The Journal of Neuroscience: The Official Journal of the Society for Neuroscience 32 (3): 787-98.

Turner, Nicholas, Kisuk Lee, Ran Lu, Jingpeng Wu, Dodam Ih, and H. Sebastian Seung. 2019. "Synaptic Partner Assignment Using Attentional Voxel Association Networks." arXiv [cs.CV]. arXiv. http://arxiv.org/abs/1904.09947.

Vaughan, Alexander G., Chuan Zhou, Devanand S. Manoli, and Bruce S. Baker. 2014. "Neural Pathways for the Detection and Discrimination of Conspecific Song in D. Melanogaster." Current Biology: CB 24 (10): 1039-49.

White, J. G., E. Southgate, J. N. Thomson, and S. Brenner. 1986. "The Structure of the Nervous System of the Nematode Caenorhabditis Elegans." Philosophical Transactions of the Royal Society of London. Series B, Biological Sciences 314 (1165): 1-340.

Wu, Chia-Lin, Meng-Fu Maxwell Shih, Jason Sih-Yu Lai, Hsun-Ti Yang, Glenn C. Turner, Linyi Chen, and Ann-Shyn Chiang. 2011. "Heterotypic Gap Junctions between Two Neurons in the Drosophila Brain Are Critical for Memory." Current Biology: CB 21 (10): 848-54.

Xu, C. S., M. Januszewski, Z. Lu, S. Takemura, and K. Hayworth. 2020. "A Connectome of the Adult Drosophila Central Brain." bioRxiv. https://www.biorxiv.org/content/10.1101/2020.01.21.911859v1.abstract.

Yamada, Daichi, Hiroshi Ishimoto, Xiaodong Li, Tsunehiko Kohashi, Yuki Ishikawa, and Azusa Kamikouchi. 2018. "GABAergic Local Interneurons Shape Female Fruit Fly Response to Mating Songs." The Journal of Neuroscience: The Official Journal of the Society for Neuroscience 38 (18): 4329-47.

Yin, Wenjing, Derrick Brittain, Jay Borseth, Marie E. Scott, Derric Williams, Jed Perkins, Chris Own, et al. 2019. "A Petascale Automated Imaging Pipeline for Mapping Neuronal Circuits with High-Throughput Transmission Electron Microscopy." bioRxiv. https://doi.org/10.1101/791889.

Zhao, Ting, Donald J. Olbris, Yang Yu, and Stephen M. Plaza. 2018. "NeuTu: Software for Collaborative, Large-Scale, Segmentation-Based Connectome Reconstruction." Frontiers in Neural Circuits 12 (November): 101.

Zheng, Zhihao, J. Scott Lauritzen, Eric Perlman, Camenzind G. Robinson, Matthew Nichols, Daniel Milkie, Omar Torrens, et al. 2018. "A Complete Electron Microscopy Volume of the Brain of Adult Drosophila Melanogaster." Cell 174 (3): 730-43.e22.

Zheng, Z., F. Li, C. Fisher, I. J. Ali, N. Sharifi, and S. Calle-Schuler. 2020. "Structured Sampling of Olfactory Input by the Fly Mushroom Body." bioRxiv. https://www.biorxiv.org/content/10.1101/2020.04.17.047167v2.abstract.

Zung, Jonathan, Ignacio Tartavull, Kisuk Lee, and H. Sebastian Seung. 2017. "An Error Detection and 
bioRxiv preprint doi: https://doi.org/10.1101/2020.08.30.274225; this version posted August 30,2020. The copyright holder for this preprint

(which was not certified by peer review) is the author/funder, who has granted bioRxiv a license to display the preprint in perpetuity. It is made available under aCC-BY-ND 4.0 International license.

Correction Framework for Connectomics." In Advances in Neural Information Processing Systems 30, edited by I. Guyon, U. V. Luxburg, S. Bengio, H. Wallach, R. Fergus, S. Vishwanathan, and R.

Garnett, 6818-29. Curran Associates, Inc. 